\title{
Titration of Syntaxin1 in Mammalian Synapses Reveals Multiple Roles in Vesicle Docking, Priming, and Release Probability
}

\author{
Marife Arancillo, ${ }^{1,2}$ Sang-Won Min, ${ }^{3}$ Stefan Gerber, ${ }^{3}$ Agnieszka Münster-Wandowski, ${ }^{4}$ Yuan-Ju Wu, ${ }^{2}$ Melissa Herman, ${ }^{2}$ \\ Thorsten Trimbuch, ${ }^{2}$ Jong-Cheol Rah, ${ }^{1}$ Gudrun Ahnert-Hilger, ${ }^{4}$ Dietmar Riedel, ${ }^{5}$ Thomas C. Südhof, ${ }^{3}$ \\ and Christian Rosenmund ${ }^{1,2}$ \\ ${ }^{1}$ Department of Molecular and Human Genetics, Baylor College of Medicine, Houston, Texas 77030, ${ }^{2}$ NeuroCure Cluster of Excellence, Charité- \\ Universitätsmedizin Berlin, 10117 Berlin, Germany, ${ }^{3}$ Department of Molecular and Cellular Physiology and Howard Hughes Medical Institute, Stanford \\ University, Stanford, California 94305, ${ }^{4}$ AG Functional Cell Biology, Institute for Integrative Neuroanatomy, Charité Centre 2 for Basic Medicine, 10115 \\ Berlin, Germany, and 5Department of Neurobiology, Max-Planck-Institute for Biophysical Chemistry, 37077 Göttingen, Germany
}

Synaptic vesicles undergo sequential steps in preparation for neurotransmitter release. Individual SNARE proteins and the SNARE complex itself have been implicated in these processes. However, discrete effects of SNARE proteins on synaptic function have been difficult to assess using complete loss-of-function approaches. We therefore used a genetic titration technique in cultured mouse hippocampal neurons to evaluate the contribution of the neuronal SNARE protein Syntaxin 1 (Stx1) in vesicle docking, priming, and release probability. We generated graded reductions of total Stx1 levels by combining two approaches, namely, endogenous hypomorphic expression of the isoform Stx1B and RNAi-mediated knockdown. Proximity of synaptic vesicles to the active zone was not strongly affected. However, overall release efficiency of affected neurons was severely impaired, as demonstrated by a smaller readily releasable pool size, slower refilling rate of primed vesicles, and lower release probability. Interestingly, dose-response fitting of Stx1 levels against readily releasable pool size and vesicular release probability showed similar $K_{\mathrm{d}}$ (dissociation constant) values at $18 \%$ and $19 \%$ of wild-type Stx1, with cooperativity estimates of 3.4 and 2.5, respectively. This strongly suggests that priming and vesicle fusion share the same molecular stoichiometry, and are governed by highly related mechanisms.

\section{Introduction}

Synaptic transmission requires the rapid, regulated exocytosis of neurotransmitter from vesicles at the presynapse. To ensure availability during activity, synaptic vesicles (SVs) undergo repeated rounds of exocytosis and endocytosis. Part of this trafficking cycle prepares vesicles for efficient merger with the plasma membrane. These steps include morphological vesicle docking to the active zone and priming, which is defined as the assembly of the molecular apparatus for action potential (AP)-evoked and

\footnotetext{
Received Jan. 12, 2013; revised Aug. 29, 2013; accepted Sept. 5, 2013.

Author contributions: M.A., G.A.-H., T.C.S., and C.R. designed research; M.A., S.-W.M., A.M.-W., Y.-J.W., M.H., and D.R. performed research; S.G., T.T., and J.-C.R. contributed unpublished reagents/analytic tools; M.A., S.-W.M., A.M.-W., Y.-J.W., M.H., G.A.-H., and D.R. analyzed data; M.A., Y.-J.W., M.H., and C.R. wrote the paper.

This research was supported by the Deutsche Forschungsgemeinschaft Research Training Group 1123 for Learning and Memory (to M.A.); and by the Neurocure Cluster of Excellence Exc257, the Collaborative Research Center 958, and the European Research Council Grant SYNVGLUT (to C.R.). We thank Ralf Nehring for assistance in designing the knock-down and rescue constructs; Benjamin Rost for design and cloning of the SynGCaMP2 lentiviral construct and for discussions on the projects; Hui Deng, Kathleen Grüttner, Metin Yenilmez, and Annegret Felies for help with cell culture; Berit Söhl-Kielczynski for help with sample preparations for microscopy; Hongmei Chen and Sabine Lenz for assistance with animal care and genotyping; Bettina Brokowski and Katja Pötschke for help with virus production, molecular biology, and DNA sequencing; and Jakob B. Sørensen for comments on the manuscript.

The authors declare no competing financial interests.

Correspondence should be addressed to Christian Rosenmund, NeuroCure Cluster of Excellence, CharitéUniversitätsmedizin Berlin, 10117 Berlin, Germany. E-mail: christian.rosenmund@charite.de.

DOI:10.1523/JNEUROSCI.0187-13.2013

Copyright $\odot 2013$ the authors $\quad 0270-6474 / 13 / 3316698-17 \$ 15.00 / 0$
}

spontaneous release before fusion (Südhof, 2004). Although key presynaptic proteins have been identified, the exact molecular interplay during docking, priming, and fusion are not fully understood.

The soluble $N$-ethylmaleimide-sensitive factor (NSF) attachment protein (SNAP) receptor (SNARE) complex is composed of the vesicle-anchored protein synaptobrevin2 (Syb2), and the plasma membrane-localized proteins syntaxin1 (Stx1) and SNAP25. Although SNARE proteins alone cannot mediate fusion in vivo without Sec1/Munc18-like protein as a partner (Südhof and Rothman, 2009), deletion of SNARE proteins in different species arrested neurotransmission. SNARE homologs are also essential for non-neuronal cellular trafficking processes (Jahn and Scheller, 2006), establishing them as highly conserved core components of the fusion machinery.

Deletion studies in mammalian systems suggest that neuronal SNARE proteins are fundamental not only for the fusion event, but also for canonical upstream steps in the exocytosis pathway. Removal of SNAP25 and Syb2 impaired evoked neurotransmitter release and severely reduced the readily releasable pool (RRP) of primed vesicles (Schoch et al., 2001; Washbourne et al., 2002; Delgado-Martínez et al., 2007). Knock-out mutants of the isoform Stx1A did not show significant differences in evoked neurotransmission (Fujiwara et al., 2006; Gerber et al., 2008), but there may be functional redundancy with another isoform, Stx $1 B$ (Bennett et al., 
1992). Interestingly, endogenous expression of an "open form" mutant of Stx1B (Dulubova et al., 1999), in the absence of Stx1A, decreased RRP but increased vesicular release probability $\left(P_{\mathrm{vr}}\right.$; Gerber et al., 2008). Thus, aside from expression, SNARE conformation can also regulate priming and fusion efficiency.

Here we provide a comprehensive analysis of the possible roles of Stx1 in preparing vesicles for efficient release, aside from having a core function in membrane fusion. Invertebrate loss-offunction mutants of Stx 1 have provided invaluable evidence for the role of SNARE proteins in fusion (Schulze et al., 1995; Ogawa et al., 1998; Saifee et al., 1998), but a complete lack of expression prohibited functional evaluation of other steps. The potentially severe effect of total Stx1 loss on neuronal development in mammals, as observed in Drosophila (Schulze et al., 1995), was another consideration. Furthermore, studies involving cleavage of Stx1 using botulinum toxins did not quantify the change in expression levels after treatment (Sakaba et al., 2005; de Wit et al., 2006). We therefore used a combination of genetic approaches in mammalian neurons, namely hypomorphic expression and RNAi-mediated knockdown (k.d.), to titrate down Stx1 to controlled amounts and study the consequences on neurotransmitter release. Using this approach, we quantified morphological and physiological correlates of docking, priming, and fusion in central synapses as a function of Stx 1 expression levels.

\section{Materials and Methods}

Generation and maintenance of mouse lines. Construction of the Stx $1 B^{y f p}$ targeting vector, generation of the mouse line, and genotyping protocols are described in detail in the Supplemental Experimental Procedures section of the study by Gerber et al. (2008). Briefly, a rat Stx1B cDNA fragment encoding residues $178-290$ was amplified by PCR, such that the stop codon was replaced by a 10 aa GS linker sequence. Enhanced yellow fluorescent protein (EYFP) was subcloned downstream of the linker sequence. The Stx1B-EYFP fusion construct was subsequently inserted in-frame into a PCR-amplified mouse Stx1B exon 7. This artificial exon 7, together with a FRT-flanked neomycin resistance gene (NEO) cassette, was inserted into intron 6 of a previously modified mouse Stx1B genomic fragment (containing the previously published L165A, E166A "open-form" mutations; Gerber et al., 2008). Assembly of the modified mouse Stx1B genomic fragment, together with overlapping fragments spanning exons $2-10$, and subcloning of the diphtheria toxin sequence into the $5^{\prime}$ end, completed the targeting vector for homologous recombination.

The Stx $1 B^{y f p}$ targeting construct was linearized and electroporated into R1 mouse embryonic stem cells. Colonies were selected for resistance to G418. Homologous recombination was verified by Southern blotting, and positive clones were expanded and injected into C57BL/6 blastocysts. Chimera mice were backcrossed, and progeny were expanded to establish colonies.

Animal maintenance. The Animal Care and Use Committees for Baylor College of Medicine, University of Texas Southwestern Medical Center, Stanford University, and the animal welfare committees of Charité Berlin and the Berlin state government approved all protocols for animal maintenance and experiments. The Stx $1 A^{-\prime-}$ mouse line was previously characterized (Gerber et al., 2008). To obtain Stx1B;Stx1A double mutants, Stx $1 A^{-/-}$mice were crossed with $S t x 1 B^{+/ y f p}$ mice. Stx $1 B^{+/ y f p}$; Stx $1 A^{+/-}$mice were selected and respectively intercrossed to obtain mice for survival studies. Control and test groups for electrophysiological, ultrastructural, and imaging experiments were obtained from littermate offspring of either sex. Genotyping for the $S t x 1 B^{y f p}$ allele by PCR used the following primers: forward, 5' -CAA GGA CCG AAT CCA GAG GCA G-3'; and reverse, 5'-GCT TTC CAA CAT GTC TTC TAG-3'. Annealing temperature was set at $60^{\circ} \mathrm{C}$. The presence of a $260 \mathrm{bp}$ band corresponded to the Stx $1 B$ wild-type allele, while a 300 bp band corresponded to the $S t x 1 B^{y f p}$ allele.

Cell culture. Primary neuronal cultures were prepared by plating embryonic day 18.5 (E18.5) or postnatal day 0 (P0) mouse-derived hip- pocampal neurons in previously made astrocyte feeder cultures. First, alkaline-treated $30 \mathrm{~mm}$ coverslips were placed in wells of culture plates and coated with $0.15 \%$ agarose. Once the agarose is dry, a cellattachment substrate consisting of acetic acid, collagen, and poly-Dlysine was applied in a dotted pattern using a custom-made rubber stamp. Astrocytes derived from mouse P1 cortices were plated 5-7 d before neurons, and grew only within acetic acid-collagen-PDL islands. For some experiments, proliferation was arrested by adding the antimitotic agent $8 \mu \mathrm{M}$ 5-fluoro-2-deoxyuridine and $20 \mu \mathrm{M}$ uridine (FUDR) before replacing astrocyte media with Neurobasal-A media, while some skipped the use of FUDR. To harvest neurons, brains were removed from E18.5 embryos or P0 newborn mice, then were carefully stripped of meninges and dissected in cold HBSS. Isolated hippocampi were incubated in $2 \mathrm{U} / \mathrm{ml}$ papain in DMEM at $37^{\circ} \mathrm{C}$. After $45 \mathrm{~min}$, papain was inactivated by incubation of tissue in a solution of albumin, trypsin inhibitor, and 5\% FCS for $10 \mathrm{~min}$. Tissue was transferred to Neurobasal-A media containing B-27 supplement (Invitrogen), $50 \mathrm{IU} / \mathrm{ml}$ penicillin and $50 \mu \mathrm{g} / \mathrm{ml}$ streptomycin, and triturated by repeated pipetting. Cells were plated with a density of 2500 or 3000 neurons per $35 \mathrm{~mm}$ well. When necessary, neurons were transduced with lentiviral constructs $18-24 \mathrm{~h}$ after plating. Cultures were incubated at $37^{\circ} \mathrm{C}$ for $9-10 \mathrm{~d}$ before starting electrophysiological recordings. For immunoblotting, protein lysates were derived from neurons plated on coverslips coated only with a collagen/poly-D-lysine cell attachment substrate.

Lentiviral constructs. Lentiviral vectors and production were based on previous work (Lois et al., 2002). HEK293T cells were cotransfected with $8 \mu \mathrm{g}$ of shuttle vector and $5 \mu \mathrm{g}$ each of the helper plasmids pVSVg and pCMVdR8.9, using the FuGENE 6 Transfection Reagent (Roche Diagnostic). Virus-containing cell culture media were collected after $48 \mathrm{~h}$ and filtered to remove cellular debris. The filtrate was distributed into aliquots, flash-frozen using liquid nitrogen, and stored at $-80^{\circ} \mathrm{C}$. The viral titer for both rescue and knock-down constructs was estimated by infecting mass cultures of hippocampal neurons and counting the number of cells expressing a fluorescent marker after $7 \mathrm{~d}$. Mouse cDNA rescue constructs of Stx1A and Stx $1 B$ were cloned into vectors that placed them under the control of the neuron-specific synapsin promoter. For RNAi knock-down experiments, a Stx1B-specific short hairpin RNA (shRNA) target sequence ( $5^{\prime}$-GAT CCC AGG CAC AAT GAG ATC ATC AAA-3') was obtained using Genscript siRNA Target Finder (http://www. genscript.com). An shRNA construct based on the target sequence was then cloned into a shuttle vector that placed them under the control of the U6 promoter. For Munc18-1 rescue experiments, Munc18-1 cDNA (kindly donated by Dr. Matthijs Verhage, Center for Neurogenomics and Cognitive Research, Amsterdam, The Netherlands) and Stx1A cDNA were cloned into a modified $2 \mathrm{~A}$ peptide-linked multicistronic vector (Kim et al., 2011) with a fluorescent marker under the control of a synapsin promoter. Stx1A or Stx1B expression levels during rescue or knockdown were assessed by immunoblotting. Protein lysates for analysis were derived from mass cultures of hippocampal neurons 13-15 d after transduction with control and experimental constructs.

Electrophysiology. During recordings, neurons were immersed in standard extracellular solution consisting of $140 \mathrm{~mm} \mathrm{NaCl}, 2.4 \mathrm{~mm} \mathrm{KCl}, 10$ mM HEPES, $10 \mathrm{~mm}$ glucose, $2 \mathrm{~mm} \mathrm{CaCl}_{2}$, and $4 \mathrm{~mm} \mathrm{MgCl}_{2}$. The patch pipette internal solution consisted of $136 \mathrm{~mm} \mathrm{KCl}, 17.8 \mathrm{~mm}$ HEPES, $1 \mathrm{~mm}$ EGTA, $0.6 \mathrm{~mm} \mathrm{MgCl}_{2}$, 4 mM ATP, $0.3 \mathrm{~mm}$ GTP, $12 \mathrm{~mm}$ creatine phosphate, and $50 \mathrm{U} / \mathrm{ml}$ phosphocreatine kinase. Both solutions were adjusted to pH 7.4 with osmolarity at $\sim 300 \mathrm{mOsm}$. Sucrose solutions for measurements of hyperosmotic release were prepared as $250,350,500$, and $1000 \mathrm{~mm}$ sucrose in standard extracellular solution. Calcium sensitivity assays were performed by application of extracellular solutions containing $0.5,2$, , or $12 \mathrm{mM} \mathrm{CaCl}_{2}$, with $1 \mathrm{mM} \mathrm{MgCl}_{2}$ each.

Solutions were applied using a fast-flow system that provides rapid exchange at time constants of $\sim 20-30$ ms (Rosenmund et al., 1995). Borosilicate glass patch pipettes were pulled using a multistep puller (P-87, Sutter Instruments) using conditions that kept pipette resistance between 2 and 3.5 M $\Omega$. Currents were recorded using Multiclamp 700B amplifier (Molecular Devices). Series resistance was compensated at $75 \%$. Data were acquired using Clampex 9.2 software (Molecular Devices) and were filtered using a low-pass Bessel filter at $3 \mathrm{kHz}$. Off-line 
analysis was performed using Axograph X (Axograph Scientific). Data summation and statistical analyses were performed using Kaleidagraph 4.0 (Synergy Software), Prism (GraphPad), and SPSS (IBM).

Whole-cell voltage-clamp recordings from autaptic neurons were obtained between 9 and $15 \mathrm{~d}$ in vitro (DIV) at room temperature $\left(\sim 25^{\circ} \mathrm{C}\right)$. To minimize variability among datasets, an approximately equal number of cells was recorded from control and experimental groups per day; data from experimental groups during 1 or 2 consecutive days of recording were normalized to the mean value of the control group on the same days. Data were collected from at least two independent cultures. EPSCs were evoked by $2 \mathrm{~ms}$ somatic depolarization from -70 to $0 \mathrm{mV}$. Release due to hypertonic stimulus was triggered by the application of sucrose solution for $3 \mathrm{~s}$ (1000 mM), $5 \mathrm{~s}(500 \mathrm{~mm}), 8 \mathrm{~s}$ ( $350 \mathrm{~mm})$, or $10 \mathrm{~s}(250 \mathrm{~mm})$. To measure the readily releasable pool of vesicles in a calciumindependent fashion, $500 \mathrm{~mm}$ sucrose solution was applied to neurons for $5 \mathrm{~s}$. A transient inward current lasting for $2-3 \mathrm{~s}$ characterizes the release of the RRP. This is followed by a steady-state current, which indicates the point when rates of refilling and release are equal. When the steady-state current is used as a baseline, the area under the transient curve indicates the total charge released by the RRP (Rosenmund and Stevens, 1996). $P_{\mathrm{vr}}$ was determined by calculating the charge ratio of mean EPSC over hyperosmotic release in $500 \mathrm{~mm}$ sucrose, after integration of the responses. To analyze short-term plasticity, EPSCs were evoked at a frequency of $10 \mathrm{~Hz}$ for $5 \mathrm{~s}$. Spontaneous release was measured by recording miniature EPSCs for $60 \mathrm{~s}$ at $-70 \mathrm{mV}$. To detect falsepositive events, $3 \mathrm{~mm}$ glutamate receptor antagonist, kynurenic acid, was applied for an equal amount of time. Traces were filtered at $1 \mathrm{kHz}$, and events were detected using a template-based algorithm in Axograph X (Clements and Bekkers, 1997). To fit $\mathrm{Ca}^{2+}$ and sucrose dose-response curves and to determine the relationships among RRP size, $P_{\mathrm{vr}}$, or pairedpulse ratio and Stx 1 expression levels, the standard Hill equation $Y=$ $M /\left[1+\left(K_{\mathrm{d}} / X\right)^{n}\right]$ was applied, where $Y$ is response amplitude, $X$ is $\mathrm{Ca}^{2+}$ or Stx1B concentrations, $M$ is the maximum response, $K_{\mathrm{d}}$ is the dissociation constant, and $n$ is cooperativity. Raw mean values are stated with the SD. Unless specified otherwise, the Student's $t$ test was performed for experiments with two groups, and ANOVA with Tukey's test was performed for experiments with three or more groups. Differences among datasets were considered significant at $p<0.05$.

Calcium imaging with SynGCaMP2. The sequence for SynGCaMP2 was cloned from an Addgene plasmid (plasmid 26124: CMV:: ratSynGCaMP2) into a lentiviral construct as described above. Autaptic neurons of specified genotypes were recorded in whole-cell patch-clamp mode, as described above. Images of SynGCaMP2 fluorescence $(128 \times 128$ pixels with $2 \times 2$ binning) were acquired with a Hamamatsu ImageEM CCD camera at a rate of $20 \mathrm{~Hz}$ (exposure time, $49 \mathrm{~ms}$ ) at $60 \times$ magnification (numerical aperture, 1.2 water). SynGCaMP2 excitation was induced by a light-emitting diode ( $470 \mathrm{~nm}$ wavelength; Prizmatix Ltd.).

SynGCaMP2 images were analyzed using ImageJ and custom macros (http://rsbweb.nih.gov/ij/plugins/). Regions of interest (ROIs) were identified from the peak fluorescence of 10 baseline-subtracted AP image sequence. ROIs were applied to the entire image series of backgroundsubtracted image sequences to determine the average baseline fluorescence $\left(F_{0}\right)$ and AP-induced change in fluorescence $(\Delta F)$ for 2, 3, 5, and 10 APs $(20 \mathrm{~Hz})$. Each trial was normalized to three $F_{0}$ images immediately preceding the AP stimulation. The peak $\Delta F / F_{0}$ was measured from these averaged traces per AP trial per cell.

RNA and protein biochemistry. Total mRNA was extracted from flashfrozen whole brains of E18.5 embryos using TRIzol reagent (Invitrogen). Reverse transcription to generate Stx1B-YFP cDNA was performed using the Transcriptor First Strand cDNA Synthesis Kit (Roche Applied Science). The open reading frame of the fusion protein was targeted using a forward primer containing a Stx1B-specific sequence $\left(5^{\prime}\right.$-TAG CTA GCA TGA AGG ATC GGA CTC AGG AG- $3^{\prime}$ ) and a reverse primer with an EYFP-specific sequence (5'-AAG GCG CGC CTT ACT TGT ACA GCT CGT CCA TG-3'). Reverse transcription PCR (RT-PCR) amplification was performed using Phusion High-Fidelity DNA polymerase (New England Biolabs) with an annealing temperature of $63^{\circ} \mathrm{C}$.

Protein lysates for immunoblot quantification were derived from mass cultures of hippocampal neurons or whole-brain lysates. Briefly, cells were lysed using $50 \mathrm{~mm}$ Tris/HCl, pH 7.6, $150 \mathrm{~mm} \mathrm{NaCl}, 1 \%$ Nonidet P-40, 0.5\% sodium deoxycholate, $250 \mu \mathrm{M}$ PMSF, and 1 tablet of Complete Protease Inhibitor (Roche Diagnostics). Proteins were separated by SDS-PAGE and transferred to nitrocellulose membranes. Membranes were then incubated with mouse monoclonal anti-Stx1A (1:5000) and rabbit polyclonal anti-Stx1B (1:1000; both from Synaptic Systems), or mouse monoclonal anti- $\beta$-tubulin III (1:1000; Sigma-Aldrich) antibodies. Primary antibodies were labeled with horseradish peroxidaseconjugated goat secondary antibodies. Detection was performed using ECL Plus Western Blotting Detection Reagents (GE Healthcare Biosciences). Ratiometric quantification of signal intensity was performed using ImageJ software.

Immunocytochemistry. Cell-surface localization of YFP was determined by incubating neuronal mass cultures with a rabbit polyclonal antibody (Ab) against GFP and GFP-derived fluorescent proteins (1:200; Synaptic Systems) at $5 \times$ concentration in PBS at $4^{\circ} \mathrm{C}$ for $45 \mathrm{~min}$. After washing, cells were subsequently fixed with $4 \%$ PFA for $10 \mathrm{~min}$ and permeabilized with PBS-Tween 20 for $5 \mathrm{~min}$. After blocking with serum in PBS-Tween 20 for 30 min, cells were incubated with mouse monoclonal antibody against Stx1 (1:1000; Synaptic Systems) for $1 \mathrm{~h}$. Primary antibodies were labeled with Alexa Fluor 555 goat anti-mouse IgG and Alexa Fluor 647 goat anti-rabbit IgG (1:500 each; Invitrogen) secondary Abs. Coverslips were mounted with ProLong Gold Antifade Reagent (Invitrogen).

To measure the degree of colocalization between surface YFP and Stx1B, images were acquired using an Andor Revolution DSD white-light confocal microscopy system (Andor Technology) at $20 \times$ optical magnification. Maximum intensity projections were created from $z$-stacks and merged using ImageJ to create colocalized images. Pearson's coefficients were calculated using built-in plugins to assess the degree of colocalization.

Stx1B expression in synapses was determined by double labeling of DIV 10 autaptic neurons with antibodies against Stx1B (rabbit polyclonal Ab, 1:2500; Synaptic Systems) and the active zone marker Bassoon (guinea pig polyclonal Ab, 1:2500; Synaptic Systems). Cells were fixed with $4 \%$ PFA, permeabilized, and blocked using the above-mentioned procedure. Primary antibodies were incubated overnight at $4^{\circ} \mathrm{C}$, and labeled with anti-guinea pig DyLight Rhodamine Red-X and anti-rabbit Alexa Fluor 647 (1:500 each; Jackson ImmunoResearch). Coverslips were mounted with Mowiol 4-88 antifade medium (Polysciences Europe).

Quantification of synaptic Stx1B levels. Stx1B was quantified by measuring fluorescence intensity in regions marked by Bassoon spots. First, images were acquired using an Andor Revolution DSD white-light confocal microscopy system (Andor Technology) at $60 \times$ optical magnification and stored as 16-bit data. Overexposure and photobleaching were avoided by checking for saturation in synaptic boutons and neuronal extensions (via built-in features of the Andor Technology iQ software), and minimized by optimizing exposure time. Consistent exposure times were used for each channel per experiment. $Z$-stacks of autaptic neurons were set with a $0.5 \mu \mathrm{m}$ interstack interval and total $z$-axis range of 3-4 $\mu \mathrm{m}$. Images were then analyzed using ImageJ software with custom plugins (http://rsb.info.nih.gov/ij/). Briefly, sum intensity projections were created from the stacks. Background subtraction and threshold adjustment were performed uniformly across all groups. Three to four regions, excluding the cell body, per neuron were chosen for analysis. Bassoon spots were selected using an intensity threshold and saved as ROIs. Bassoon and Stx1B fluorescence intensities in their respective channels were measured using the same ROIs. Mean Stx1B expression levels in synapses were calculated as the ratio of average Stx1B intensity over average Bassoon intensity. These ratios were normalized against the control group.

Two-dimensional electron microscopy. DIV 10 neuronal mass cultures were fixed at room temperature for $45 \mathrm{~min}$ using a freshly prepared solution of 4\% PFA (Electron Microscopy Sciences), supplemented with $0.1 \%$ glutaraldehyde (Fluka Chemie) in $0.1 \mathrm{~m}$ phosphate buffer, $\mathrm{pH}$ 7.4. Following fixation, neurons were rinsed in phosphate buffer and then fixed in phosphate-buffered $0.1 \% \mathrm{OsO}_{4}$ for $45 \mathrm{~min}$ at $4{ }^{\circ} \mathrm{C}$ in the dark. After several washes in phosphate buffer and $\mathrm{ddH}_{2} \mathrm{O}$, neurons were stained with $2 \%$ aqueous uranyl acetate for $45 \mathrm{~min}$ at $4{ }^{\circ} \mathrm{C}$ in the dark, rinsed again in $\mathrm{ddH}_{2} \mathrm{O}$, dehydrated in graded ethanol $(30,50,70,90,2 \times$ 
$100 \%$ ), infiltrated overnight in a 1:1 mixture of hydroxypropyl methacrylate (Sigma-Aldrich Chemie) and of Epon-812 resin (Sigma-Aldrich Chemie), and finally left in pure resin overnight at room temperature. Neurons were embedded on gelatin capsules (Plano) containing freshly prepared resin (coverslips on top with the cells side down) and polymerized for $20 \mathrm{~h}$ at $60^{\circ} \mathrm{C}$. Coverslips were removed using liquid nitrogen, and regions of the block containing the neurons were remounted for sectioning. Ultrathin sections $(60 \mathrm{~nm})$ were cut parallel to the plane of the substrate using a Reichert Ultracut and were mounted on 200-mesh formvar-coated nickel grids (Plano). Thin sections were stained with uranyl acetate (Merck) and lead citrate, and were examined using a Zeiss EM 900 microscope equipped with a digital camera (1K Slow-Scan CCD Camera, Proscan). Neurons from three separate culture preparations were qualitatively examined, and one was selected for quantitative analysis.

Quantitative analysis of ultrastructural parameters was performed to determine the differences between individual genotypes. Micrographs of each genotype were taken at a magnification of $30,000 \times$. Images were imported into the iTEM software package (Olympus Soft Imaging Solutions). Only excitatory synapses identified by their asymmetric junctions were included in datasets. The area of each synapse was determined by outlining the membrane by using the closed polygon tool of the iTEM software package. All SVs within one bouton were included in the dataset, while nonvesicular structures such as cisternae or endosomes were excluded. The length of the presynaptic active zone and postsynaptic densities (PSDs) of a particular synapse were measured by using an open polyline tool of the iTEM software package. The thickness of PSDs in cell culture at DIV 10 is less developed and was not calculated. For each of the ultrastructural features analyzed, all groups were initially compared using a one-way ANOVA.

Electron microscopic tomography. Samples were prepared using two slightly different methods of chemical fixation. Cells were immersed either in $4 \%$ PFA and $0.25 \%$ glutaraldehyde in $0.1 \mathrm{~m}$ phosphate buffer, $\mathrm{pH}$ 7.4 , or in $2.5 \%$ glutaraldehyde in $0.1 \mathrm{~m}$ cacodylate buffer, $\mathrm{pH} 7.4$. Postfixation and staining was performed with $0.5 \% \mathrm{OsO}_{4}$ and $2 \%$ uranyl acetate after the first fixation method, and $1 \% \mathrm{OsO}_{4}$ and $1 \%$ uranyl acetate after the second. Samples were dehydrated using ethanol and embedded in hydroxypropyl methacrylate plus Epon-812 resin (1:1). Thin sections $(70-150 \mathrm{~nm})$ were counterstained with lead citrate and examined using a Philips CM 120 BioTwin transmission electron microscope.

Tilt series from 210-nm-thick sections from Epon-embedded cells were recorded on the Philips CM120 electron microscope at 27,500× magnification on a TVIPS $2 \mathrm{k} \times 2 \mathrm{k}$ slow-scan CCD camera in unbinned mode. Series were taken from $-64.5^{\circ}$ to $64.5^{\circ}$ in intervals using a $3^{\circ}$ Saxton scheme and were calculated using an Etomo package (http://bio3d. colorado.edu/). Distances of the vesicles to the plasma membrane and models were calculated using $3 \mathrm{dmod}$ software. Segmentation of the plasma membrane was performed using linetrack, wherein the vesicles represented only points, to visualize the distribution of the vesicle in the cluster. Analysis of mean and variance of vesicle distances to the release site (within $\sim 80 \mathrm{~nm}$ ) showed no significant difference between neurons of identical genotypes treated with slightly differing fixatives $\left[S t \times 1 B^{+/+}\right.$; Stx1 $1 A^{-1-}: 13.50 \mathrm{~nm}(\mathrm{SD}, 12.49 \mathrm{~nm})$ vs $13.24 \mathrm{~nm}(\mathrm{SD}, 10.13 \mathrm{~nm})$; + k.d.: $16.76 \mathrm{~nm}(\mathrm{SD}, 13.83 \mathrm{~nm})$ vs $13.28 \mathrm{~nm}(\mathrm{SD}, 9.72 \mathrm{~nm}) ; S_{\text {Sx } 1 B^{y f p} / y f p}$; Stx1 $1 A^{-1-}: 18.41 \mathrm{~nm}(\mathrm{SD}, 13.82 \mathrm{~nm})$ vs $16.71 \mathrm{~nm}(\mathrm{SD}, 10.05 \mathrm{~nm})$; + k.d.: $19.38 \mathrm{~nm}(\mathrm{SD}, 17.21 \mathrm{~nm})$ vs $17.61 \mathrm{~nm}(\mathrm{SD}, 10.90 \mathrm{~nm})]$. Therefore, datasets from the two experiments were pooled.

\section{Results}

A mouse line endogenously expressing a Stx1B-YFP fusion construct as a model for altered Stx1B expression levels

The study derived from an initial attempt to produce a knock-in mouse line expressing fluorescent protein-tagged Stx1B to study SNARE proteins using imaging approaches. The $S t x 1 B^{y f p}$ mouse line was generated by targeting a replacement cassette containing the $S t x 1 B$ allele fused with EYFP cDNA at the $3^{\prime}$ end (Stx1B $\left.B^{y f p}\right)$ into the endogenous locus, via homologous recombination in ES cells (Fig. 1A; Materials and Methods). To prevent potential interference of EYFP with SNARE interactions within the synaptic terminal, the fusion protein was designed such that the fluorescent molecule is extracellular and connected to the C-terminal transmembrane domain of Stx1B via a linker sequence (Fig. 1B, top, wild-type Stx1B, bottom, Stx1B-YFP). Expression of the Stx $1 B^{y f p}$ knock-in allele was verified by RT-PCR amplification of the open reading frame from $S t x 1 B^{+/ y f p}$ mRNA (Fig. 1C) and sequencing. Interestingly, when Stx1B-YFP levels were quantified by immunoblotting of brain lysates, the $\sim 70 \mathrm{kDa}$ fusion protein showed a significantly lower level of expression compared with wild-type Stx1B (Fig. 1D). It was not determined whether the expression of Stx1B-YFP is reduced at the transcriptional, translational, or post-translational level. Nevertheless, immunocytochemistry for extracellularly localized YFP was performed to determine whether the translated fusion protein was properly oriented in the plasma membrane or positioned in a manner that could interfere with function. YFP was detected at the surface of unpermeabilized $S t x 1 B^{y f p / y f p}$ neurons, while wild-type neurons expressing an intracellular fluorescent marker for lentiviral transduction showed little or no signal within the dynamic range (Fig. $1 E)$. In addition, surface YFP shows a high degree of colocalization with Stx1B (Pearson's coefficient $=0.920$; SD $=0.021$ ). Together, these results indicate that endogenously expressed Stx1B-YFP is properly oriented in the membranes but is present in severely reduced amounts compared with wild type. The fusion protein can be detected in the hippocampi and cerebella of $S t x 1 B^{y f p / y f p}$ mice using antibodies against the fluorescent tag (Fig. $1 F)$.

We then determined the expression profile of $S t x 1 B^{y f p / y f p}$ mice for other synaptic proteins. Levels of Syb2, SNAP25, tomosyn, complexin 1 (Cplx-A), synapsin 1 (Syn1), NSF protein, rabphilin (Rph), Rab3A, synaptophysin 1 (Syp1), synaptotagmin 1 (Syt), Rim1A, ELKS, liprin, and synaptojanin 1 (Synj1) were not significantly different in Stx1 $B^{y f p / y f p}$ compared with wild-type controls (Fig. 1G; Table 1). However, there was a $\sim 25 \%$ decrease in Munc18-1 expression in $S t x 1 B^{y f p / y f p}$ lysates, which was not observed in Stx $1 A^{-/-}$mice, indicating that Stx1B stabilizes Munc18-1. This is not an unexpected finding, as it is consistent with the well known physical and functional interactions between the syntaxin and Sec1/ Munc18 family of proteins (Rizo and Südhof, 2012).

Whole-cell voltage-clamp recordings from autaptic hippocampal neurons show that average peak amplitudes of EPSCs between wild-type (11.3 nA; SD, $9.7 \mathrm{nA} ; n=38)$ and $S t x 1 B^{y f p / y f p}$ neurons (11.1 nA; SD, $10.6 \mathrm{nA} n=36$ ) are not significantly different $\left(t_{(72)}=0.51, p=0.61\right.$; data not shown $)$. Short-term plasticity behavior was analyzed by high-frequency stimulation at $10 \mathrm{~Hz}$ for $5 \mathrm{~s}$. Both wild-type and Stx $1 B^{y f p / y f p}$ AP-evoked responses initially undergo facilitation, and then depression to reach steady-state, without significant differences in normalized amplitudes (data not shown). In addition to evoked responses, the RRP of fusion-competent vesicles was measured by rapid application of hyperosmotic solution (Materials and Methods; Rosenmund and Stevens, 1996). The RRP charges of wild-type neurons ( $1.84 \mathrm{nC} ; \mathrm{SD}, 1.79 \mathrm{nC} ; n=39)$ and Stx $1 B^{y f p / y f p}$ neurons $(2.09 \mathrm{nC} ; \mathrm{SD}, 1.76 \mathrm{nC} ; n=37)$ are not significantly different from each other $\left(t_{(74)}=0.44, p=0.66\right.$; data not shown). Taken collectively, these results indicate that although $S t x 1 B$ expression is reduced in $S t x 1 B^{y f p / y f p}$ hippocampal neurons, neurotransmitter release is not significantly affected.

Although there are no significant changes in hippocampal neurotransmission in culture, and the gross morphology of new- 
A genomic Syntaxin $1 \mathrm{~B}$ - clones $\mathrm{N} \longrightarrow \mathrm{B}-\mathrm{Bg} \quad \mathrm{E}-\mathrm{B}-\mathrm{Bg}-\mathrm{Bg}$

Syntaxin 1B - gene

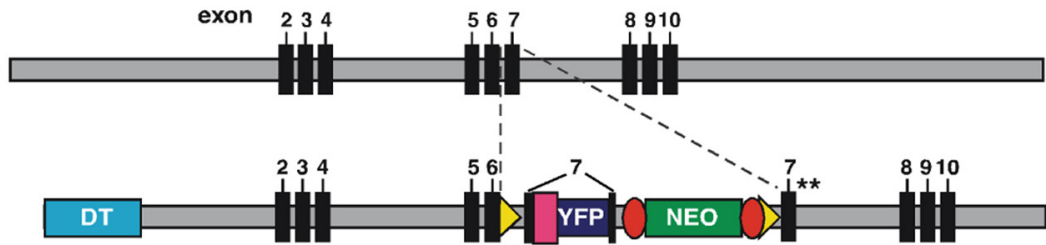

Knockin vector

DT

YFP-Knockin gene

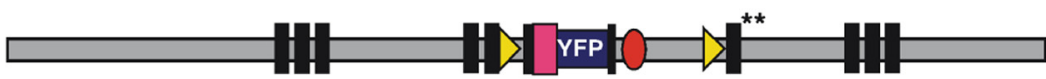

B

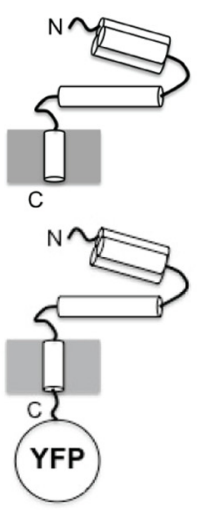

C loxP FRT CDNA-fragment

$2 \mathrm{~Kb}$
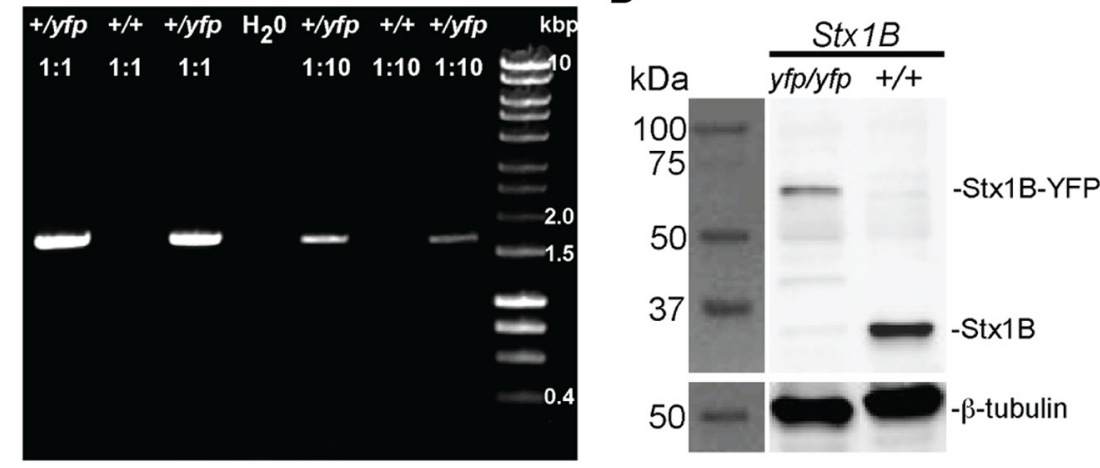

$\mathbf{E}$
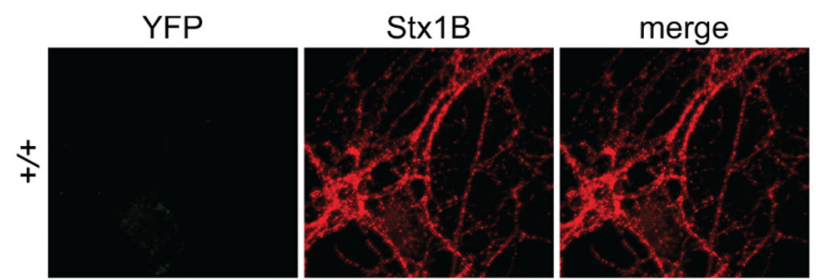

\section{G}

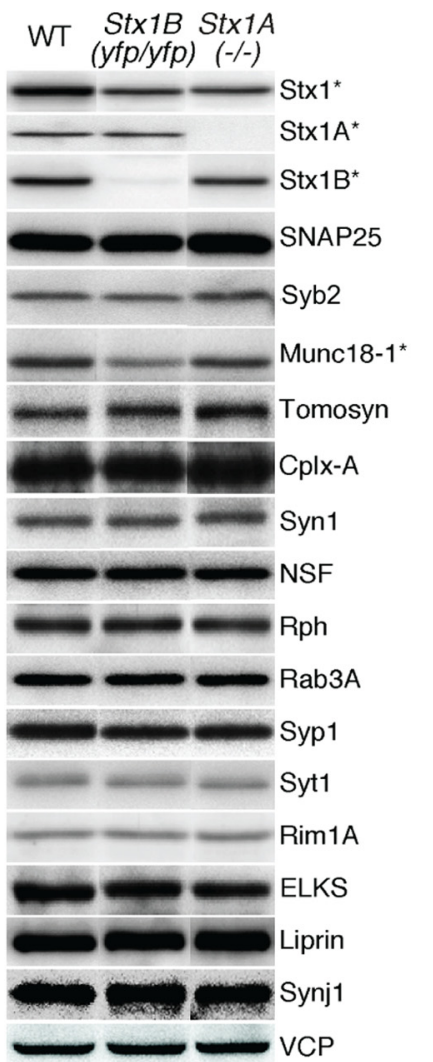

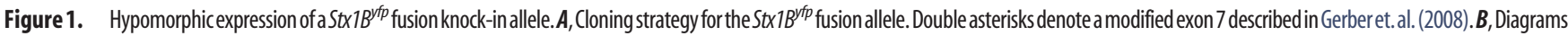
depicting the orientations of plasma membrane-anchored wild-type (top) and EYFP-fused Stx1B (bottom). C, RT-PCR amplification of a full $\sim 1.6 \mathrm{~kb}$ transcript encoded by the Stx $1 B^{\text {yrp }}$ allele from Stx $1 B^{+/ y f p}$

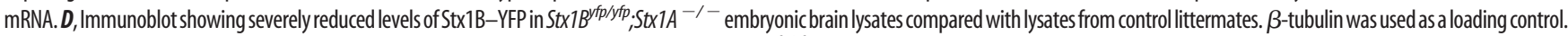
$\boldsymbol{E}$, Immunofluorescent staining of hippocampal mass cultures for extracellularly localized YFP. Stx $7 B^{\text {yp } / y f p}$, but not wild-type controls transduced with intracellular fluorescent protein, is positive for surface expression of YFP. Scale bar, $10 \mu \mathrm{m}$. F, Peroxidase immunostaining of Stx1B-YFP fusion protein in sagittal sections of P14 hippocampus and cerebellum. Antibodies to synaptotagmin 1 (Syn1) and Rab3A were used as positive controls, while PBS corresponds to negative control. DG, Dentate gyrus; MF, hippocampal mossy fiber synapse. G, Immunoblot showing expression levels of SNARE proteins, Munc18-1, and other synaptic proteins in wild-type, Stx $1 B^{\text {yppyfyp }}$, and Stx $1 A^{-/-}$P14 mice. Valosin-containing protein (VCP) was used as a loading control. Asterisks mark proteins with significant changes in expression levels. 
Table 1. Quantification of synaptic protein expression levels from immunoblots

\begin{tabular}{|c|c|c|c|c|c|}
\hline & $1 A^{W T} 1 B^{W T}$ & $1 A^{\mathrm{WT}} 1 \mathrm{~B}^{\mathrm{YFP}}$ & $1 \mathrm{~A}^{\mathrm{K} 0} 1 \mathrm{~B}^{\mathrm{WT}}$ & $\begin{array}{l}p \text { value: } \\
A^{\text {WT }} B^{\text {WT }} \text { vs } \\
A^{\text {WT }} B^{\text {YFP }}\end{array}$ & $\begin{array}{l}p \text { value: } \\
A^{W T} B^{W T} \text { vs } \\
A^{K 0} B^{W T}\end{array}$ \\
\hline Syntaxin-1A T3748* & $100 \pm 10.8$ & $83.6 \pm 6.0$ & $2.6 \pm 0.1$ & 0.154 & 0.007 \\
\hline Syntaxin-1B 4191 & $100 \pm 7.6$ & $2.7 \pm 0.1$ & $110 \pm 2.1$ & 0.003 & 0.105 \\
\hline Syntaxin-1 HPC-1 & $100 \pm 11.4$ & $41.2 \pm 3.1$ & $70.2 \pm 1.1$ & 0.004 & 0.034 \\
\hline Syntaxin-1 I-379 & $100 \pm 6.1$ & $67.5 \pm 3.7$ & $70.5 \pm 3.2$ & 0.004 & 0.005 \\
\hline Syntaxin-1 U6250 & $100 \pm 4.3$ & $89.7 \pm 2.8$ & $39.9 \pm 3.0$ & 0.058 & 0.003 \\
\hline SNAP-25 & $100 \pm 7.7$ & $110 \pm 6.0$ & $106 \pm 6.7$ & 0.255 & 0.505 \\
\hline Synaptobrevin-2 & $100 \pm 7.6$ & $102 \pm 10.9$ & $102 \pm 7.2$ & 0.861 & 0.827 \\
\hline Munc18-1 monoclonal & $100 \pm 4.7$ & $45.8 \pm 3.3$ & $81.8 \pm 6.9$ & 0.0001 & 0.053 \\
\hline Munc18-1 J370 & $100 \pm 12.8$ & $74.4 \pm 5.6$ & $97.8 \pm 6.3$ & 0.072 & 0.855 \\
\hline Munc18-1 J371 & $100 \pm 8.0$ & $70.9 \pm 3.6$ & $96.0 \pm 8.2$ & 0.014 & 0.700 \\
\hline Munc18-1 K329 & $100 \pm 14.7$ & $70.3 \pm 1.1$ & $91.9 \pm 1.5$ & 0.056 & 0.510 \\
\hline Munc18-1 P592 & $100 \pm 12.1$ & $71.9 \pm 6.8$ & $91.6 \pm 9.0$ & 0.058 & 0.511 \\
\hline Complexin-A & $100 \pm 3.6$ & $96.4 \pm 4.2$ & $92.5 \pm 8.0$ & 0.462 & 0.364 \\
\hline Synaptotagmin 1 & $100 \pm 10.5$ & $89.6 \pm 6.0$ & $93.9 \pm 5.0$ & 0.346 & 0.571 \\
\hline Rab3A & $100 \pm 6.2$ & $100.7 \pm 3.6$ & $98.7 \pm 9.8$ & 0.911 & 0.901 \\
\hline Rabphilin & $100 \pm 5.5$ & $95.4 \pm 4.9$ & $103 \pm 12.8$ & 0.474 & 0.795 \\
\hline Synapsin & $100 \pm 10.2$ & $97.6 \pm 5.5$ & $96.1 \pm 11.2$ & 0.820 & 0.767 \\
\hline Synaptophysin 1 & $100 \pm 4.9$ & $87.2 \pm 6.8$ & $87.8 \pm 9.2$ & 0.114 & 0.233 \\
\hline NSF & $100 \pm 7.4$ & $105 \pm 8.8$ & $116 \pm 16.6$ & 0.602 & 0.310 \\
\hline Synaptojanin 1 & $100 \pm 6.3$ & $89.1 \pm 6.1$ & $97.1 \pm 5.5$ & 0.204 & 0.683 \\
\hline $\operatorname{RIM} 1 \alpha$ & $100 \pm 4.6$ & $102 \pm 4.9$ & $94.7 \pm 6.1$ & 0.718 & 0.417 \\
\hline ELKS & $100 \pm 8.7$ & $116 \pm 11.9$ & $104.2 \pm 6.6$ & 0.266 & 0.655 \\
\hline Liprin & $100 \pm 3.5$ & $93.2 \pm 8.3$ & $99.9 \pm 4.2$ & 0.377 & 0.983 \\
\hline Synaptogyrin-1 & $100 \pm 8.2$ & $92.2 \pm 9.2$ & $112 \pm 6.5$ & 0.476 & 0.245 \\
\hline VGluT 1 & $100 \pm 16.1$ & $100 \pm 10.9$ & $102.7 \pm 11.1$ & 0.999 & 0.878 \\
\hline CASK & $100 \pm 6.4$ & $109 \pm 2.9$ & $104 \pm 5.1$ & 0.182 & 0.571 \\
\hline
\end{tabular}

*Numerical codes indicate different antibodies.

born homozygous mice is similar to that of wild-type mice, juveniles eventually stopped gaining weight and died at 3 weeks (Fig. $2 A, B)$. These results are in contrast to mice lacking the isoform Stx1A, which were generally healthy, had normal lifespans, and exhibited basal excitatory synaptic transmission similar to wildtype mice (Fujiwara et al., 2006; Gerber et al., 2008). We then tested functional redundancy between $S t x 1 A$ and $S t x 1 B$ by generating $S t x 1 B^{y f p / y f p} ;$ Stx $1 A^{-/-}$double-mutant mice. In this case, lethality manifested at an even earlier time point compared with Stx $1 B^{y f p / y f p}$ mice, as demonstrated by death of double homozygotes very shortly after birth (Fig. 2B). Examination of E18.5 embryos revealed gross morphological abnormalities such as smaller size, curved spine, and deformed head and neck structures compared with control littermates (Fig. 2C). Such developmental defects were similar to findings in other SNARE protein knockouts (Schoch et al., 2001; Washbourne et al., 2002). Meanwhile, heterozygote littermates $\left(S t \times 1 B^{+/ y f p} ;\right.$ Stx $\left.1 A^{-/-}\right)$are physically indistinguishable from $S t \times 1 B^{+/+} ; S t x 1 A^{-/-}$controls, are fertile at the normal breeding age, and have normal lifespans. Though a full Stx $1 A^{-/-} ;$St $x 1 B^{-/-}$double knockout is necessary for verification, these findings demonstrate that mammalian Stx $1 A$ and $S t x 1 B$ are functionally redundant during embryonic development. On the other hand, wild-type Stx $1 B$ is essential and sufficient for postnatal survival.

\section{Reduced Stx 1 expression diminished AP-evoked and spontaneous neurotransmitter release}

We then used the reduced expression of the Stx1B-YFP protein (Fig. 1D) as an opportunity to study synaptic properties and neurotransmitter release efficiency under hypomorphic conditions. In doing so, we aimed to circumvent a complete block of neu- rotransmitter release associated with the complete loss of SNARE protein.

While synaptic transmission in $S t x 1 B^{y f p / y f p}$ neurons was not perturbed, neurotransmitter release in $S t x 1 B^{y f p / y f p} ;$ Stx $1 A^{-1-}$ neurons showed severe impairments. EPSC amplitude was dramatically decreased by $\sim 80 \%$ in $\operatorname{Stx} 1 B^{y f p / y f p}$;Stx $1 A^{-/-}$neurons (0.49 nA; SD, $1.5 \mathrm{nA} ; n=69)$ compared with controls (3.7 nA; $\mathrm{SD}, 6.3 \mathrm{nA} ; n=83 ; F_{(2,180)}=48, p<0.0001$; Fig. $\left.2 D, E\right)$, and EPSC rise times were longer by $\sim 30 \%\left(\right.$ Stx $1 B^{+/+} ;$Stx $1 A^{-/-}$controls: $1.9 \mathrm{~s} ; \mathrm{SD}, 0.59 \mathrm{~s} ;$ Stx $1 B^{+/ y f p} ;$ Stx $1 A^{-/-}: 1.6 \mathrm{~s} ; \mathrm{SD}, 0.38 \mathrm{~s}$; Stx $1 B^{y f p / y f p} ;$ Stx $1 A^{-l-}: 2.5 \mathrm{~s} ; \mathrm{SD}, 0.88 \mathrm{~s} ; F_{(2,109)}=15.03, p<$ 0.0001 ; Fig. $2 F)$, strongly indicating impairments in evoked release efficiency. In addition, miniature EPSC frequency was severely reduced by $\sim 70 \%$ in $S t x 1 B^{y f p / y f p}$;Stx $1 A^{-1-}$ double mutants $\left(0.64 \mathrm{~s}^{-1}\right.$; SD, $\left.0.43 \mathrm{~s}^{-1} ; n=66\right)$, compared with $S t \times 1 B^{+/+}$; Stx $1 A^{-1-}$ controls $\left(3.7 \mathrm{~s}^{-1} ; \mathrm{SD}, 3.8 \mathrm{~s}^{-1} ; n=60 ; t_{(124)}=6.5, p<\right.$ 0.0001 ; Fig. $2 G, H)$. The mean amplitude and charge of mEPSC events are slightly reduced $\left(S t \times 1 B^{+/+} ; S t x 1 A^{-/-}: 29.9 \mathrm{pA} ; \mathrm{SD}, 9.5\right.$ $\mathrm{pA} ; n=59$; and $110 \mathrm{fC} ; \mathrm{SD}, 37.1 \mathrm{fC} ; n=59 ;$ Stx $1 B^{y f p / y f p}$; Stx $1 A^{-1-}: 24.2 \mathrm{pA} ; \mathrm{SD}, 9.4 \mathrm{pA} ; n=65$; and $92.7 \mathrm{fC}$; SD, $50.0 \mathrm{fC}$; $n=65 ; t_{(122)}=2.7, p=0.0070$ and $t_{(122)}=2.3, p=0.026$, data not shown). Evoked release in $S t x 1 B^{+/ y f p} ;$ Stx $1 A^{-1-}$ neurons (3.3 $\mathrm{nA}$; SD, $7.5 \mathrm{nA} ; n=31)$ is not significantly different from controls (Fig. $2 D, E$ ). These results suggest that AP-evoked and spontaneous forms of neurotransmitter release are sensitive to levels of Stx 1 ; one wild-type copy of Stx 1 is sufficient to achieve normal levels of neurotransmitter release, but levels of Stx1 below a certain threshold begin to cause impairments.

Stx1-deficient neurons have a smaller readily releasable pool The availability of fusion-competent vesicles is a key determinant of neurotransmitter release. To determine whether reduced Stx1B levels change RRP characteristics, we measured the size and refilling speed of vesicles that are available for fusion in $S t x 1 B^{y f p / y f p}$; Stx $1 A^{-/-}$neurons, independent of external calcium concentrations. Stx $1 B^{y f p / y f p} ;$ Stx $1 A^{-/-}$neurons $(0.30 \mathrm{nC}$; SD, $0.38 \mathrm{nC} ; n=$ 70 ) showed a $\sim 65 \%$ reduction in RRP charge compared with Stx $1 B^{+/+} ;$Stx $1 A^{-/-}$controls (1.07 nC; SD, $\left.1.24 \mathrm{nC} ; n=85\right)$, while Stx $1 B^{+/ y f p} ;$ Stx $1 A^{-1-}$ showed no significant changes $(1.39$ $\mathrm{nC}$; SD, $0.89 \mathrm{nC} ; n=32 ; F_{(2,186)}=28, p<0.0001$; Fig. $\left.3 A, B\right)$. To determine the calcium-independent refilling rate of the RRP, a paired sucrose assay was performed. In this experiment, neurons were treated with two consecutive applications of hypertonic solution at increasing time intervals to measure the fraction of the RRP refilled after a given period. Data were fitted into a single exponential equation to derive time constants $(\tau)$, which were used to evaluate refilling rates. The average $\tau$ for refilling in Stx $1 B^{y f p / y f p}$;Stx $1 A^{-/-}$mutants was $4.1 \mathrm{~s}$, while $\tau$ for $S t x 1 B^{+/+}$; Stx $1 A^{-1-}$ controls was $1.5 \mathrm{~s}$. The rate of RRP refilling in Stx 1 hypomorphs is therefore significantly slower by $\sim 63 \%$ compared with controls (two-way ANOVA, $F_{(2,143)}=4.68, p=0.01$; Fig. $3 C, D)$. Taken collectively, these results indicate that $S t x 1$ is a critical factor for vesicle priming.

\section{Stx1 expression levels are critical for vesicle fusogenicity}

We then asked whether vesicles from the readily releasable pool of $S t x 1 B^{y f p / y f p} ;$ Stx $1 A^{-1-}$ neurons fuse as efficiently as vesicles from $S t \times 1 B^{+/+} ;$Stx $1 A^{-/-}$controls and addressed this question in several ways. First, we measured the overall efficiency of APevoked release by a $\mathrm{Ca}^{2+}$ dose-response assay and measurement of vesicular release probability. $\mathrm{Ca}^{2+}$ dose-response assay was performed by recording EPSCs at different calcium concentrations (Fig. 4A). To control for rundown and cell-to-cell variabil- 


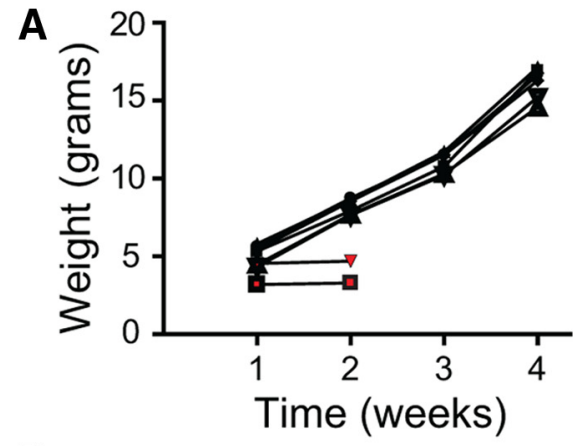

B $\bar{\pi}$

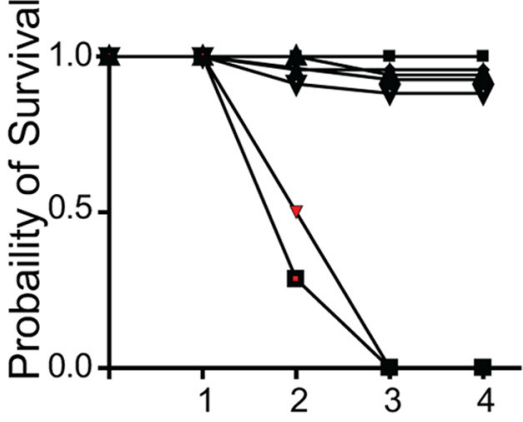

C

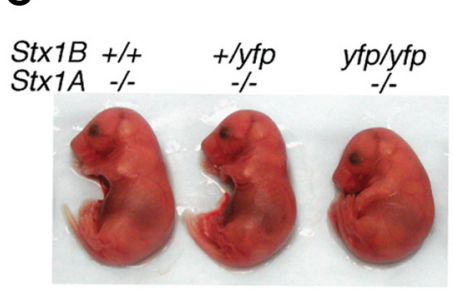

E

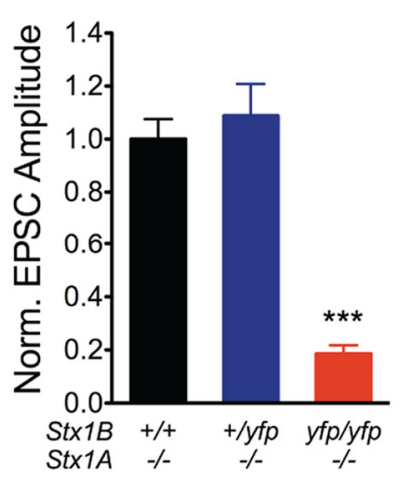

G

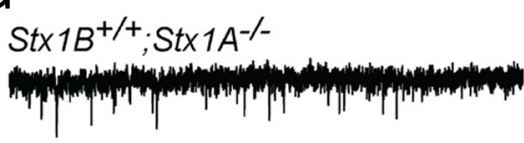

Stx $1 B^{y f p / y f p} ; S t \times 1 A^{-/-}$

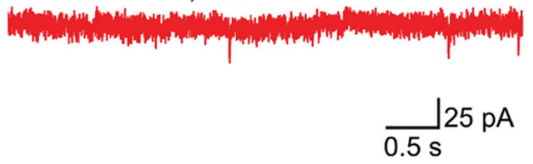

Stx1B(+/+);

Stx1A(+/+) $(n=10)$

$\operatorname{Stx} 1 B(+/+)$;

$\operatorname{Stx} 1 A(+/-)(\mathrm{n}=24)$

$\triangle \operatorname{Stx} 1 B(+/+)$;

$\operatorname{Stx} 1 A(-/)(\mathrm{n}=17)$

Stx1B(+/yfp);

Stx $1 A(+/+)(\mathrm{n}=27)$

Stx1B $(+/ y f p)$;

Stx1A(+/-) $(\mathrm{n}=44)$

$\boldsymbol{\nabla}^{S t \times 1 B(+/ y f p) ;}$

Stx1A(-/- $)(\mathrm{n}=34)$

Stx1B(yfp/yfp);

Stx $1 A(+/+)(n=10)$

Stx1B(yfp/yfp);

$\operatorname{Stx} 1 A(+/-)(\mathrm{n}=7)$

ity, test responses were normalized to average EPSCs in base external solution that were recorded before and after treatment. Normalized test responses were then normalized to the response at $12 \mathrm{~mm}$ $\mathrm{Ca}^{2+}$ (maximum concentration). In addition, EPSCs were measured in both ascending and descending orders of calcium concentration from an equal number of cells. The normalized values were fitted into a standard Hill equation to plot doseresponse curves (Fig. $4 B$ ). The $S t x 1 B^{y f p / y f p}$; Stx $1 A^{-/-}$dose-response curve is shifted markedly toward higher $\mathrm{Ca}^{2+}$ concentrations [0.5 mM: $0.10 \mathrm{nA}(\mathrm{SD}, 0.15 \mathrm{nA}), n=$ 25; 2 mM: $1.1 \mathrm{nA}$ (SD, $1.3 \mathrm{nA}), n=25 ; 4$ mM: $1.4 \mathrm{nA}$ (SD, $1.5 \mathrm{nA}), n=26 ; 12 \mathrm{~mm}$ : $1.6 \mathrm{nA}(\mathrm{SD}, 1.3 \mathrm{nA}), n=26$ ] relative to Stx $1 B^{+/+} ;$Stx $1 A^{-/-}$controls $[0.5 \mathrm{~mm}$ : $0.61 \mathrm{nA}$ (SD, $0.82 \mathrm{nA}$ ), $n=31 ; 2 \mathrm{~mm}: 3.2$ $\mathrm{nA}(\mathrm{SD}, 3.6 \mathrm{nA}), n=29 ; 4 \mathrm{mM}: 3.9 \mathrm{nA}$ (SD, $4.1 \mathrm{nA}), n=27 ; 12 \mathrm{~mm}: 5.18 \mathrm{nA}$ (SD, $5.4 \mathrm{nA}), n=29 ; F$ test, $F_{(3,166)}=8.3, p<$ 0.0001; Fig. $4 B$ ]. The apparent k.d. was significantly increased by $\sim 50 \%$ in $S t x 1 B^{y f p / y f p}$; Stx $1 A^{-1-}$ neurons (2.3 mM; SD, $0.15 \mathrm{~mm}$; $n=20)$ compared with controls $(1.6 \mathrm{mM}$; $\mathrm{SD}, 0.13 \mathrm{~mm} ; n=23 ; t_{(166)}=3.9, p=$ $0.0002)$, while the Hill coefficient $(n)$ was not affected [2.6 (SD, 0.48) vs 2.4 (SD, 0.47); $\left.t_{(166)}=0.30 ; p=0.76\right]$. Together, these data indicate reduced apparent sensitivity to extracellular $\mathrm{Ca}^{2+}$ in $\mathrm{Stx} 1 B^{y f p / y f p} ; \mathrm{Stx} 1 A^{-/-}$ neurons.

$P_{\mathrm{vr}}$ is another measure of release efficiency. It is the average likelihood that a fusion-competent vesicle will be released due to an action potential. We calculated $P_{\mathrm{vr}}$ as the ratio between the charge of the evoked release and the RRP charge. In Stx $1 B^{+/+} ;$Stx $1 A^{-/-}$control neurons, this probability is $5.3 \%$ (SD, 3.9\%). Heterozygotes have a $P_{\mathrm{vr}}$ of $5.3 \%$ (SD, 3.5\%), while Stx $1 B^{y f p / y f p} ;$ Stx $1 A^{-/-}$neurons have a $P_{\mathrm{vr}}$ of only $1.9 \%$ (SD, 2.6\%). The $\sim 60 \%$

$\leftarrow$

Stx $1 B^{y f p / y f p}$ show impaired growth and development before death at 3 weeks. $B$, Survival curves for the $S t x 1 B^{y f p}$ mouse line. Stx $1 A^{+/+} ; S t x 1 B^{y f p / y f p}$ and $S t x 1 A^{+/-} ; S t x 1 B^{\text {yfp/yfp }}$ die postnatally after 3 weeks. Stx $1 A^{-/-} ;$Stx $1 B^{\text {yfp/yfp }}$ are not shown because they die at birth. $C$, Gross morphological abnormalities in $S t x 1 B^{y f p / y f p} ; S t x 1 A^{-1-}$ embryos compared with Stx $1 B^{+/ y f p} ; S t \times 1 A^{-1-}$ and Stx $1 B^{+/+} ; S t \times 1 A^{-/-}$littermates at E18.5. D, Sample traces of EPSCs from Stx $1 B^{+/+}$; Stx $1 A^{-1-}$ (black), Stx $1 B^{+/ y f p} ;$ Stx $1 A^{-1-}$ (blue), and Stx $1 B^{\text {yfp/yfp; }} ;$ Stx $1 A^{-\prime-}$ (red) autaptic neurons. E, Normalized summary plot of EPSC peak amplitudes $\left({ }^{* * *} p<0.0001\right)$. $\boldsymbol{F}$, Normalized summary plot of EPSC rise times $\left({ }^{* * *} p<0.0001\right)$. $G$, Sample traces of $\mathrm{mEPSCs}$ recorded at $-70 \mathrm{mV}$ from Stx $1 B^{+/+} ; S t \times 1 A^{-1-}$ (black) and Stx $1 B^{\text {yfp/yfp }} ;$ Stx $1 A^{-1-}$ (red) neurons. $\boldsymbol{H}$, Normalized summary plot of mEPSC frequency $\left({ }^{* * *} p<0.0001\right)$.

Figure 2. $S t x 1 B^{y f p / y f p} ; S t x 1 A^{-1-}$ double mutants show premature lethality, gross morphological defects, and diminished $\mathrm{Ca}^{2+}$-evoked and spontaneous release. $A$, Weight curves for the $S \operatorname{tx} 1 B^{y f p}$ mouse line. Stx $1 A^{+/+} ; \operatorname{Stx} 1 B^{y f p / y f p}$ and $S t x A^{+/-}$; 
A

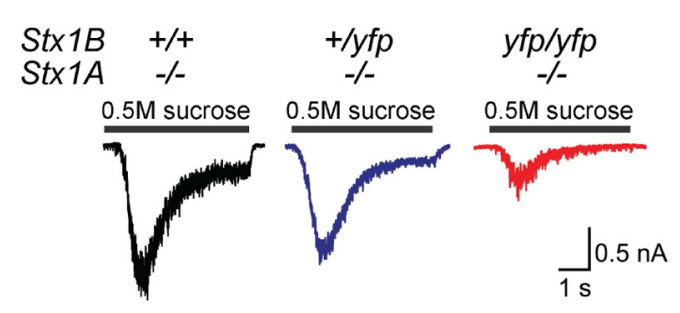

C

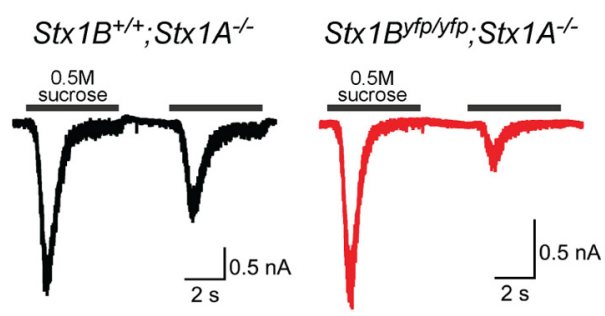

B

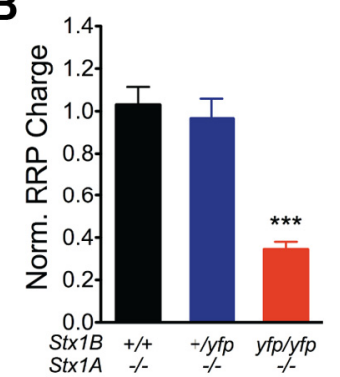

D

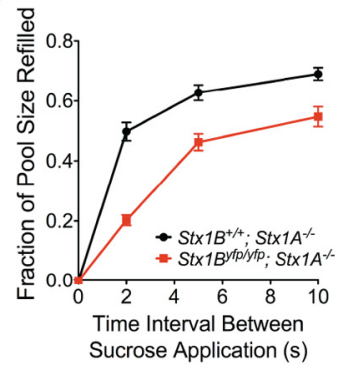

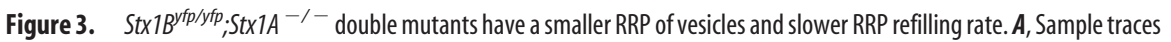

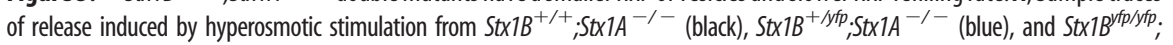
$S t \times 1 A^{-1-}$ (red) autaptic neurons. $\boldsymbol{B}$, Normalized summary plot of RRP charge $\left({ }^{* * *} p<0.0001\right)$. C, Refilling of the RRP as measured by paired applications of $0.5 \mathrm{M}$ sucrose in $\mathrm{Stx} 1 \mathrm{~B}^{+/+} ; \mathrm{Stx} 1 \mathrm{~A}^{-1-}$ (black) and $\mathrm{Stx} 1 \mathrm{~B}^{\mathrm{yfp} / \mathrm{yfp} ;} ; \mathrm{Stx} 1 \mathrm{~A}^{-1-}$ (red). D, Fraction of the RRP refilled 2,5, and $10 \mathrm{~s}$ after the first hyperosmotic stimulus $\left({ }^{* *} p<0.001\right)$.

decrease in $P_{\mathrm{vr}}\left(F_{(2,181)}=27.1, p=0.012\right.$; Fig. $\left.4 C\right)$ indicates impaired release efficiency upon severe reduction in Stx1 levels. We then determined short-term plasticity behavior to verify the observed changes in $P_{\mathrm{vr}}$. During high-frequency stimulation, neurons with "high" release probability generally exhibit depression, while those with "low" release probability show facilitation. Consistent with this phenomenon, control neurons consistently showed depression during a $10 \mathrm{~Hz}$ train of action potentials, while $S t x 1 B^{y f p / y f p}$; Stx $1 A^{-/-}$neurons displayed strong facilitation (Fig. 4D). In summary, decreased apparent $\mathrm{Ca}^{2+}$ sensitivity and lower $P_{\mathrm{vr}}$ in $S t x 1 B^{y f p / y f p} ; S t x 1 A^{-1-}$ neurons indicate that a reduction in $S t x 1$ expression significantly decreases vesicle fusion efficiency.

AP-evoked release can be considered a product of several factors, namely calcium influx into the terminals and excitationsecretion coupling, which in turn can be affected by the efficiency of the calcium-sensing apparatus and the intrinsic vesicular fusogenicity. Therefore, although $\mathrm{Ca}^{2+}$ dose-response curves are conventionally used to evaluate putative changes in the $\mathrm{Ca}^{2+}$ sensing apparatus, the contribution of the intrinsic fusion machinery in regulating release efficiency is equally a ratelimiting factor for apparent sensitivity to calcium. This has been demonstrated by the roles of Stx1 conformation and Complexin on regulating fusion kinetics (Gerber et al., 2008; Xue et al., 2010). We then tested for $\mathrm{Ca}^{2+}$-independent vesicular fusogenicity, which can be probed by assessing release as a function of hypertonicity. Briefly, neurons were stimulated with different concentrations of sucrose to gradually increase hypertonicity, and then the charge released under each concentration (Fig. $4 E$ ) was normalized to the release at maximum concentration (1.0 M sucrose) and plotted as a sigmoidal dose-response curve. The k.d. of Stx $1 B^{y f p / y f p} ;$ Stx $1 A^{-/-}$neurons $(0.36 \mathrm{M}$; SD, $0.0065 \mathrm{M} ; n=31)$ is $\sim 20 \%$ higher compared with k.d. of $\operatorname{Stx} 1 B^{+/+}$; Stx $1 A^{-1-}$ controls $(0.30 \mathrm{M}$; SD, $0.0064 \mathrm{M} ; n=33)$, indicating that they have a lower sensitivity to hyperosmotic stimulation ( $F$ test, $F_{(2,339)}=9.4, p<0.0001$; Fig. $\left.4 F\right)$. Overall, these data suggest a decreased vesicular fusogenicity of $S t x 1 B^{y f p / y f p} ; S t x 1 A^{-/-}$neurons.

Vesicle fusogenicity in the absence of $\mathrm{Ca}^{2+}$-triggered release can also be assessed by measuring spontaneous release rate. Spontaneous release rate was calculated by dividing mEPSC frequency (Fig. $2 \mathrm{H}$ ) by the number of vesicles in the RRP (RRP charge over mean mEPSC charge). Conceptually, this determines the fraction of the RRP released spontaneously per second. St $x 1 B^{y f p / y f p} ;$ Stx $1 A^{-1-}$ neurons have a $42 \%$ lower spontaneous release rate $\left(0.00034 \mathrm{~s}^{-1}\right.$; SD, $\left.0.00032 \mathrm{~s}^{-1} ; n=51\right)$ compared with controls $\left(0.00059 \mathrm{~s}^{-1} ; \mathrm{SD}\right.$, $\left.0.00068 \mathrm{~s}^{-1} ; n=50\right)$ consistent with a decreased vesicular fusogenicity $\left(t_{(99)}=\right.$ 2.342, $p=0.0212$; Fig. $4 G$ ).

Taken collectively, our results suggest that Stx1 deficiency impairs neurotransmitter release efficiency by decreasing vesicular release probability, mainly through a lowered vesicle fusogenicity.

\section{Changes in presynaptic calcium influx do not account for changes in synaptic release}

Another possible explanation for the reduced calcium sensitivity in $S t x 1 B^{y f p / y f p} ;$ Stx $1 A^{-1-}$ neurons was reduced AP-evoked $\mathrm{Ca}^{2+}$ influx through voltage-gated calcium channels. To test this hypothesis, we performed imaging experiments on genetically encoded $\mathrm{Ca}^{2+}$ indicator fused to synaptophysin, SynGCaMP2, which has been used previously to show relative changes in presynaptic $\mathrm{Ca}^{2+}$ influx between conditions (Zhao et al., 2011). We found that induction of APs in Stx $1 B^{y f p / y f p} ;$ Stx $1 A^{-\prime-}$ neurons expressing SynGCaMP2 resulted in a clear increase in fluorescence (Fig. 5A). To compare $\mathrm{Ca}^{2+}$ influx between genotypes, regardless of SynGCaMP2 expression level, we compared the average $\Delta F$ normalized to $F_{0}$ (Fig. $5 B, C$ ). Overall, two-way ANOVA analysis showed no effect of genotype on the AP-evoked SynGCaMP2 signals (Fig. 5C). It should be noted, however, that the Bonferroni post-test reported a significant difference $\left({ }^{*} p<0.05\right)$ at 10 APs between $S t x 1 B^{+/+} ; S t x 1 A^{-/-}$and $S t x 1 B^{y f p / y f p} ; S t x 1 A^{-/-}$ neurons, but not between any other stimulus number or genotype combination. Though the slopes of the linear regression fits differed significantly between the three groups $\left[{ }^{*} p=0.03\right.$;

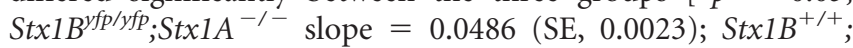
Stx $1 A^{-/-}$slope $=0.0570(\mathrm{SE}, 0.0015) ;$ Stx $1 B^{+/ y f p} ;$ Stx $1 A^{-/-}$ slope $=0.0524$ (SE 0.0002)], we do not believe this difference could account for the reduction of EPSC amplitude (Fig. 5D) or the increase in paired-pulse ratio (Fig. $5 E$ ) in $S t x 1 B^{y f p / y f p}$; Stx $1 A^{-/-}$neurons compared with $S t \times 1 B^{+/+} ; \operatorname{Stx} 1 A^{-/-}$or Stx $1 B^{+/ y f p} ;$ Stx $1 A^{-/-}$neurons.

\section{Knockdown of Stx1B-YFP enhanced the reduction in release efficiency}

If Stxl expression levels modulate the readily releasable pool and fusion efficiency, then we expect an enhancement of the Stx $1 B^{y f p / y f p} ;$ Stx $1 A^{-/-}$phenotype upon further reduction of Stx $1 B^{y f p}$ expression. To test this hypothesis, an shRNA construct that reduces the expression of both wild-type Stx1B and Stx1BYFP fusion proteins was expressed in $S t x 1 B^{y f p / y f p} ;$ Stx $1 A^{-/-}$via 

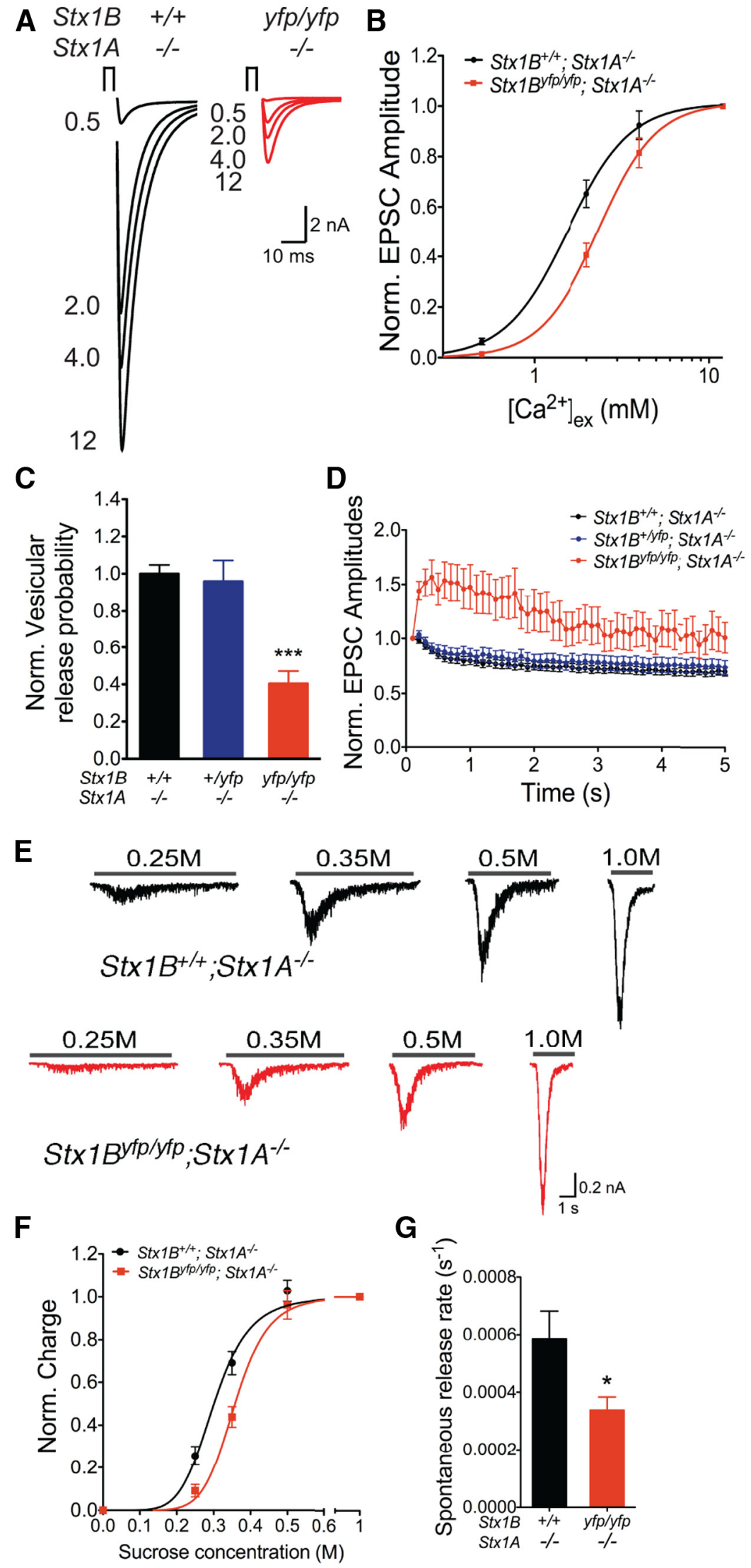

Figure 4. Vesicle fusogenicity is impaired in $S t x 1 B^{y f p / y f p} ; S t x 1 A^{-1-}$ neurons. $A$, Sample traces of evoked responses from Stx $1 B^{+1+} ;$ Stx $1 A^{-1-}$ (black) and Stx $1 B^{\text {yfp/yff }}$.Stx $1 A^{-1-}$ (red) neurons at different external $\mathrm{Ca}^{2+}$ concentrations. B, Ca ${ }^{2+}$ doseresponse curves for $S t x 1 B^{+/+} ; S t x 1 A^{-1-}$ (black) and $S t x 1 B^{y f p / y f p} ; S t x 1 A^{-/-}$(red) neurons. C, Normalized summary plot of $P_{\mathrm{vr}}$ measured as the ratio of the EPSC charge and RRP charge, in Stx $1 B^{+/+} ;$Stx $1 A^{-\prime-}$ (black), Stx $1 B^{+/ y f p} ;$ Stx $1 A^{-/-}$(blue), and lentivirus vector. Transduction of the shRNA construct in neuronal mass cultures reduces levels of both Stx1B (36\% reduction compared with untransduced Stx $1 B^{+/+} ;$Stx $1 A^{-/-}$neurons; $F_{(2,5)}=$ $12.42, p=0.0115)$ and Stx1B-YFP expression $(62 \%$ reduction compared with untransduced $S t x 1 B^{y f p / y f p} ;$ Stx $1 A^{-\prime-}$ neurons; $\left.F_{(2,5)}=21.86, p=0.0034\right)$, but not that of Stx1A (11\% reduction compared with untransduced Stx $1 B^{+/+} ;$Stx $1 A^{-/-}$neurons; $\left.F_{(2,5)}=0.1008, p=0.8990\right)$, demonstrating the specificity of the knockdown (Fig. 6A).

Reduction of Stx1B-YFP expression by knockdown (k.d.) dramatically decreased the evoked responses of $S t x 1 B^{y f p / y f p}$; Stx $1 A^{-1-}$ neurons by $\sim 80 \%(0.12 \mathrm{nA}$; $\mathrm{SD}, 0.20 \mathrm{nA} ; n=32$ ) compared with untransduced $(0.82 \mathrm{nA} ; \mathrm{SD} 1.2 \mathrm{nA} ; n=32)$ or vector-transduced neurons $(0.53 \mathrm{nA}$; $\mathrm{SD}, 0.66 \mathrm{nA} ; n=27 ; F_{(3,116)}=72.42, p<$ 0.0001 ; Fig. $6 B, D$ ). RRP size was reduced to a similar degree by $\sim 80 \%\left(\operatorname{Stx} 1 B^{+/+}\right.$; Stx1 $1 A^{-/-}$: $899.0 \mathrm{pC} ; \mathrm{SD}, 974 \mathrm{pC} ; n=28$; Stx $1 B^{y f p / y f p} ; S_{1 x} 1 A^{-1-}: 300.5 \mathrm{pC} ; \mathrm{SD}, 239$ $\mathrm{pC} ; n=27 ; \operatorname{Stx}_{1 B^{y f p} / y f p} ; \operatorname{Stx} 1 A^{-/-}+$vector: 357.8 pC; SD, 358 pC; $n=27$; Stx $1 B^{y f p / y f p}$; Stx1 $1 A^{-/-}+$k.d.: $79.2 \mathrm{pC} ;$ SD, $135 \mathrm{pC} ; n=$ $32 ; F_{(3,109)}=24.23, p<0.0001$; Fig. $\left.6 C, E\right)$. Interestingly, Stx $1 B^{y f p / y f p} ; S t x 1 A^{-1-}$ neurons transduced with the knock-down construct did not lead to further significant changes in $P_{\mathrm{vr}}$ compared with the untransduced hypomorphs $\left(\operatorname{Stx}_{1} 1 B^{+/+} ; \operatorname{St} \times 1 A^{-/-}\right.$: $5.3 \% ; \mathrm{SD}, 6.7 \% ; n=28 ; \operatorname{Stx} 1 B^{y f p / y f p}$; Stx1 $\mathrm{A}^{-1-}: 1.4 \%$; SD, $2.3 \% ; n=32$; Stx $1 B^{\text {yfp } / y f p} ;$ Stx $1 A^{-1-}+$ vector: $1.7 \%$; SD, $2.7 \% ; n=27 ; \operatorname{Stx}_{1} B^{y f p / y f p} ; S_{t x} 1 A^{-1-}$ + k.d.: $0.96 \%$; SD, $1.8 \% ; n=36 ; F_{(3,110)}=$ 25.76, $p<0.0001$; Fig. $6 F$ ). Consistent with $P_{\mathrm{vr}}$ results, shRNA-transduced Stx $1 B^{y f p}$ /yfp $; S t x 1 A^{-1-}$ neurons facilitated to a similar degree as untransduced or vector-transduced neurons (Fig. 6G). In summary, knockdown of Stx1B-YFP expression, in a genetic background with already reduced Stx1 expression, further diminishes evoked release and RRP, without significantly affecting $P_{\mathrm{vr}}$

\section{Knockdown of wild-type Stx $1 B$} mimicked hypomorphic effects We analyzed neurotransmitter release in Stx $1 B^{+/+} ; S \operatorname{St} 1 A^{-/-}$neurons transduced

\section{$\leftarrow$}

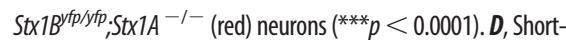

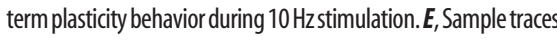
of release induced by different (hyperosmotic) sucrose concentrations. $\boldsymbol{F}$, Dose-response curves from hypertonic sucrose stimulation. $G$, Spontaneous release rate, calculated as the fraction of the RRP released spontaneously per second $\left({ }^{*} p<0.05\right)$. 


\section{A}

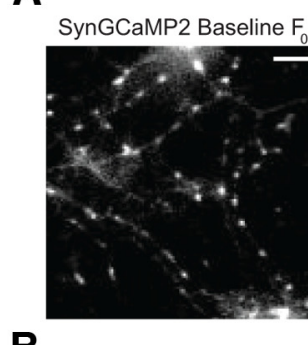

B

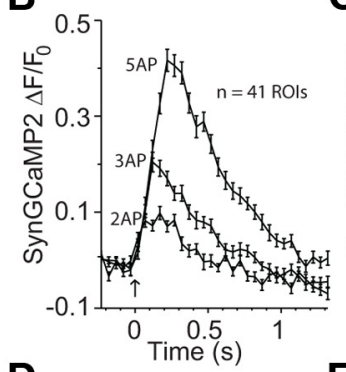

D

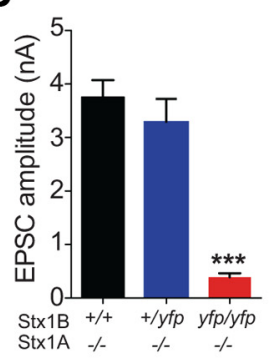

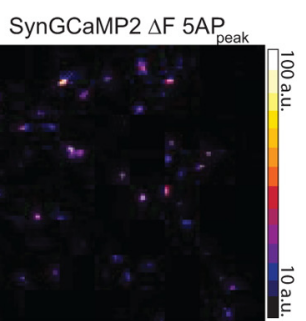

C

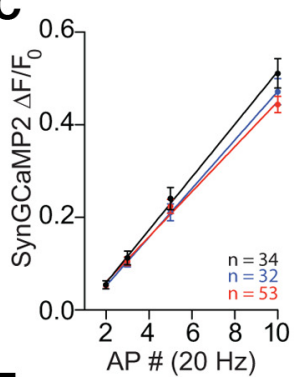

$\mathbf{E}$

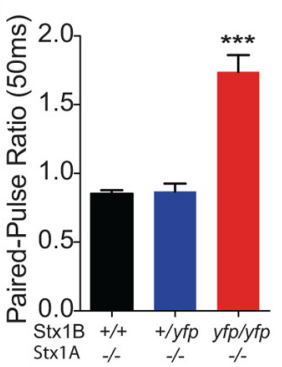

Figure 5. Presynaptic $\mathrm{Ca}^{2+}$ influx is not altered in $S t x 1 B^{\text {yfp/yfp }} ; S t x 1 A^{-1-}$ neurons. $\boldsymbol{A}$, Example baseline fluorescence (left; average of three images; $F_{0}$ ) and peak 5 AP fluorescence

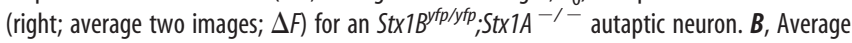
$\Delta F / F_{0}$ for ROls selected from neuron shown in $\boldsymbol{A}(n=41)$ in response to 2,3 , and $5 \mathrm{AP}$ at $20 \mathrm{~Hz}$. Arrow indicates initiation of stimulation. $C$, Average $\Delta F / F_{0}$ responses per cell for $S t x 1 B^{\text {yfp/yfp }}$; Stx $1 A^{-1-}$ (red; $n=35$ cells) and control (black; Stx $1 B^{+/+} ;$Stx $1 A^{-/-}$and Stx $1 B^{+/ y f p}$; Stx $1 A^{-1-}$ pooled; $n=33$ ) for 2, 3, 5, and $10 \mathrm{AP}$ at $20 \mathrm{~Hz}$. D, E, Data were fitted with a linear regression analysis (red and black lines, respectively). Average paired-pulse ratio ( $\boldsymbol{D} ; 50 \mathrm{~ms}$ interstimulus interval; $\left.{ }^{* * *} p<0.0001\right)$ and EPSC amplitude $\left(\boldsymbol{E}^{* * * *} p<0.0001\right)$ for Stx1 ${ }^{\text {yfp/yfp; }}$ Stx1A ${ }^{-/-}$and control cells from C. All error bars represent SEM.

with Stx1B-specific shRNA as an alternative approach to analyzing Stx $1 B^{y f p / y f p} ;$ Stx $1 A^{-1-}$ neurons. St $x 1 A^{-1-}$ neurons transduced with the knock-down construct showed an $\sim 70 \%$ reduction in evoked release compared with untransduced and vector-transduced controls $\left(\right.$ Stx $1 B^{+/+} ; S t x 1 A^{-/-}: 3.0 \mathrm{nA}$; SD, 2.6 $\mathrm{nA} ; n=39 ; \operatorname{Stx}_{1 B^{+/+}} ;$Stx $1 A^{-/-}+$vector: $2.8 \mathrm{nA} ; \mathrm{SD}, 3.3 \mathrm{nA} ; n=$ $37 ; S_{x} 1 B^{+/+} ;$Stx $1 A^{-/-}+$k.d.: $1.1 \mathrm{nA} ; \mathrm{SD}, 1.9 \mathrm{nA} ; n=41 ; F_{(2,114)}=$ $10.8, p<0.0001$; Fig. $7 A, B)$. In addition, the RRP was reduced by $\sim 60 \% \quad\left(\right.$ Stx $1 B^{+/+} ;$Stx $1 A^{-/-}: 323.1 \mathrm{pC}$, SD 315.5, $n=39$; Stx $1 B^{+/+} ;$Stx $1 A^{-/-}+$vector: $333.8 \mathrm{pC}, \mathrm{SD} 399.4, n=37$; Stx $1 B^{+/+} ;$Stx $1 A^{-/-}+$k.d.: 101.7 pC; SD, 165.8 pC; $n=42$; $F_{(2,115)}=9.8, p<0.0001$; Fig. $\left.7 C, D\right), P_{\mathrm{vr}}$ is lower by $\sim 50 \%$

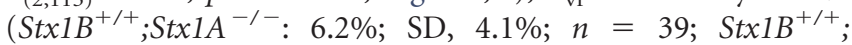

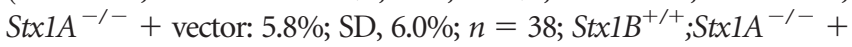
k.d.: $5.1 \%$; SD, 3.9\%; $n=39 ; F_{(2,113)}=4.5, p<0.05$; Fig. $\left.7 E\right)$, and facilitation occurred upon high-frequency stimulation (Fig. $7 F$ ). These results were analogous to $S t x 1 B^{y f p / y f p} ; S t x 1 A^{-/-}$phenotypes, suggesting that low expression levels, rather than the presence of the YFP domain in $S t x 1 B^{y f p}$ knock-in mice, led to reduced release efficiency.

We quantified synaptic density to determine whether there were impairments in central synapse number and distribution

upon Stx1 reduction. To ensure that all autaptic neurons analyzed were excitatory, vesicular glutamate transporter 1 (VGluT1) was used as a synaptic marker. The number of VGluT1 puncta over dendritic length, which was measured using the staining pattern of antibodies against microtubule-associated protein 2 (MAP2), was used as an indicator of synaptic density (Chao et al., 2007). The number of puncta per $100 \mu \mathrm{m}$ dendrite length was comparable between transduced and untransduced Stx $1 A^{-/-}$neurons $\left(\right.$Stx $1 B^{+/+} ;$Stx $1 A^{-/-}: 22 / 100 \mu \mathrm{m}$; SD, 6.9/

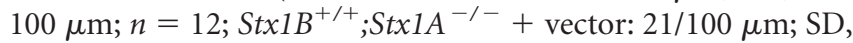
9.4/100 $\mu \mathrm{m} ; n=13 ;$ Stx $1 B^{+/+} ;$Stx $1 A^{-/-}+$k.d.: $24 / 100 \mu \mathrm{m} ; \mathrm{SD}$, $9.2 / 100 \mu \mathrm{m} ; n=14 ; F_{(2,37)}=0.3588, p=0.7009$; Fig. $\left.7 G, H\right)$. This suggests that defects in neurotransmission were not likely caused by defects in synaptic development, but rather by the release machinery itself.

\section{Exogenous expression of either Stx1A or Stx1B, but not} Munc18-1, is sufficient to rescue neurotransmitter release A decrease in Munc18-1 levels was observed in Stx1-deficient neurons (Fig. $1 G$ ). Given the important role of Munc18-1 in synaptic release and vesicle priming (Rizo and Südhof, 2012), this finding raises the possibility that the associated loss of Munc18-1 is the underlying reason for reduced evoked and spontaneous release, vesicle priming, and release probability. We therefore performed a rescue experiment by lentivirally expressing Stx1A, Stx1B, or Munc18-1 in Stx $1 B^{y f p / y f p} ;$ Stx $1 A^{-/-}$neurons, and Stx1A or Munc18-1 in Stx1 $A^{-/-}$neurons with a Stx1B-specific knockdown construct (Fig. 6A). Lentiviral expression of either Stx1 wild-type isoform restored EPSC amplitudes, RRP charges, and vesicular release probability to a degree comparable to $S t x 1 B^{+/+}$; Stx $1 A^{-/-}$controls, while neurons transduced with Munc18-1 were not significantly different from $S t x 1 B^{y f p / y f p} ; S t x 1 A^{-1-}$ neurons (EPSC amplitudes: $F_{(4,202)}=8.3, p<0.0001$; RRP Charges: $F_{(4,210)}=10.1, p<0.0001 ; P_{\mathrm{vr}}: F_{(4,210)}=9.0, p<0.0001$; Fig. $8 A-C)$, verifying that the expression of either Stx $1 A$ or $S t x 1 B$ is sufficient for normal synaptic transmission in hippocampal neurons. In $S t x 1 B^{+/+} ; S t x 1 A^{-/-}$neurons with knockdown, exogenous expression of Munc18-1 alone also did not rescue synaptic release, in contrast to expression of Stx1A (EPSC amplitudes: $F_{(3,119)}=10.6, p<0.0001$; RRP charges: $F_{(3,120)}=10.8, p<$ $0.0001 ; P_{\mathrm{vr}}: F_{(3,116)}=4.8, p<0.05$; Fig. $\left.8 D-F\right)$. Together, our results demonstrate that increasing Munc18-1 expression alone does not bypass Stx1 deficiency and rescue impaired neurotransmitter release. In addition, restoring Stxl expression is sufficient for synaptic function, indicating that the defects in release, vesicle priming, and release probability seen in Stx1-deficient neurons are directly related to the loss of the molecule.

\section{Synaptic vesicle distribution upon reduction of Stx1 expression}

We observed severe impairments in functionally defined priming and release probability upon reduction of Stx 1 expression, but an effect on vesicle docking is unclear. To analyze docking, we generated three-dimensional projections of synapses by tomography. Compared with conventional transmission electron microscopy (TEM), tomography increases the resolution in the $z$-axis to give a more precise representation of vesicle distribution from the plasma membrane. We assessed docking by measuring mean vesicle distance from the plasma membrane. Stx $1 B$ knockdown in $S t x 1 B^{+/+} ; S t x 1 A^{-/-}$neurons did not cause significant changes in cumulative vesicle distribution compared with untransduced controls, while $S t x 1 B^{y f p / y f p} ; S t x 1 A^{-1-}$ neurons with or without knockdown showed a small but significant increase in 
the fraction of vesicles residing farther away from the membrane (KolmogorovSmirnov $z$ test $=1.8, p=0.003$ for both; Fig. $9 A, B)$. Mean vesicle distance within a range of $40 \mathrm{~nm}$ from the plasma membrane is increased by $\sim 25 \%$ in $S t x 1 B^{y f p / y f p}$; Stx $1 A^{-/-}$neurons with or without knockdown $\left(\right.$ Stx $1 B^{+/+} ;$Stx $1 A^{-/-}: 13.3$ $\mathrm{nm}$; SD, $9.4 \mathrm{~nm} ;$ Stx $1 B^{+/+} ;$Stx $1 A^{-/-}+$ k.d.: $14.6 \mathrm{~nm}$; SD, $9.6 \mathrm{~nm}$; Stx1B $B^{y f p / y f p}$; Stx1 $1 A^{-/-}$: $16.7 \mathrm{~nm} ; \mathrm{SD}, 10.4 \mathrm{~nm}$; Stx $1 B^{y f p / y f p} ;$ Stx $1 A^{-1-}+$ k.d.: 16.5; SD, $10.1 \mathrm{~nm}$; Kruskall-Wallis $t$ test $=11.25$, $p=0.011$; Fig. 9C).

We determined whether Stxl levels were indeed significantly reduced in the samples analyzed by measuring total fluorescence intensity of Stx1B among the different groups. In this experiment, knockdown in $\operatorname{Stx} 1 B^{+/+} ;$Stx $1 A^{-/-}$neurons resulted in a $56 \%$ reduction (SD, $26 \%$, while Stx $1 B^{y f p / y f p} ;$ Stx $1 A^{-/-}$neurons with or without knockdown showed $76 \%(\mathrm{SD}, 12 \%)$ and $67 \%(\mathrm{SD}, 12 \%)$ reductions, respectively. The weaker reduction in $S t x 1 B^{+/+} ;$Stx $1 A^{-/-}$neurons with knockdown compared with $S t x 1 B^{y f p / y f p}$; Stx $1 A^{-1-}$ neurons could explain the lack of impairments in vesicle distribution in the former group. Overall, these data suggest that Stx 1 is involved in docking, but levels have to be reduced by $\sim 70 \%$ to start observing changes in vesicle positions.

Meanwhile, changes in synaptic morphology were evaluated by conventional TEM. Presynaptic terminal size $\left(\right.$ Stx $1 B^{+/+}$;

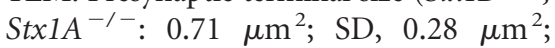
Stx $1 B^{+/+}$;Stx $1 A^{-\prime-}+$ k.d.: $0.63 \mu \mathrm{m}^{2}$; SD, $0.28 \mu \mathrm{m}^{2}$; Stx $1 B^{y f p / y f p} ;$ Stx $1 A^{-/-}: 0.59$ $\mu \mathrm{m}^{2} ; \quad \mathrm{SD}, \quad 0.36 \mu \mathrm{m}^{2} ; \quad$ Stx $1 B^{y f p / y f p}$; Stx $1 A^{-1-}+$ k.d.: $0.66 \mu \mathrm{m}^{2}$; SD, 0.66 $\mu \mathrm{m}^{2}$; Fig. 9D) and active zone length $\left(\right.$ Stx $1 B^{+/+} ;$Stx $1 A^{-/-}: 796 \mathrm{~nm}$; SD, $321 \mathrm{~nm}$; Stx $1 B^{+/+} ;$Stx $1 A^{-/-}+$k.d.: $913 \mathrm{~nm} ; \mathrm{SD}$, $385 \mathrm{~nm} ;$ Stx1B $1 B^{y f / y f p} ;$ Stx $1 A^{-/-}: 955 \mathrm{~nm}$; SD, $341 \mathrm{~nm}$; Stx $1 B^{y f p / y f p} ;$ Stx $1 A^{-/-}+$k.d.: 956 nm; SD, 308 nm; Fig. 9G) were comparable among all four groups $\left(F_{(3,116)}=\right.$ $0.92, p=0.43$; and $F_{(3,116)}=1.5, p=0.22$, respectively; $n=30$ synapses), indicating no changes in synaptic architecture upon reduction of Stx 1 levels. The total number of synaptic vesicles and synaptic vesicle density were not significantly different in $\operatorname{St} x 1 B^{+/+} ;$St $x 1 A^{-/-}$transduced with the knock-down construct, while these were slightly decreased in $S t x 1 B^{y f p / y f p} ;$ Stx $1 A^{-/-}$neurons with and without Stx $1 B$ knockdown $\left(\right.$ Stx $1 B^{+/+} ;$Stx $1 A^{-/-}: 124$ vesicles; SD 60 vesicles; $88 / 0.5$ $\mu \mathrm{m}^{2}$; SD, 20/0.5 $\mu \mathrm{m}^{2}$; Stx $1 B^{+/+} ;$Stx $1 A^{-/-}+$k.d.: 104 vesicles; SD, 53 vesicles; 82/0.5 $\mu^{2}$; SD, 27/0.5 $\mu \mathrm{m}^{2}$; Stx $1 B^{y f p / y f p}$; Stx $1 A^{-/-}: 78$ vesicles; SD, 58 vesicles; and $66 / 0.5 \mu \mathrm{m}^{2} ; \mathrm{SD}, 22 /$ $0.5 \mu \mathrm{m}^{2} ;$ Stx $1 B^{y f p / y f p} ;$ Stx $1 A^{-/-}+$k.d.: 82 vesicles; SD, 28
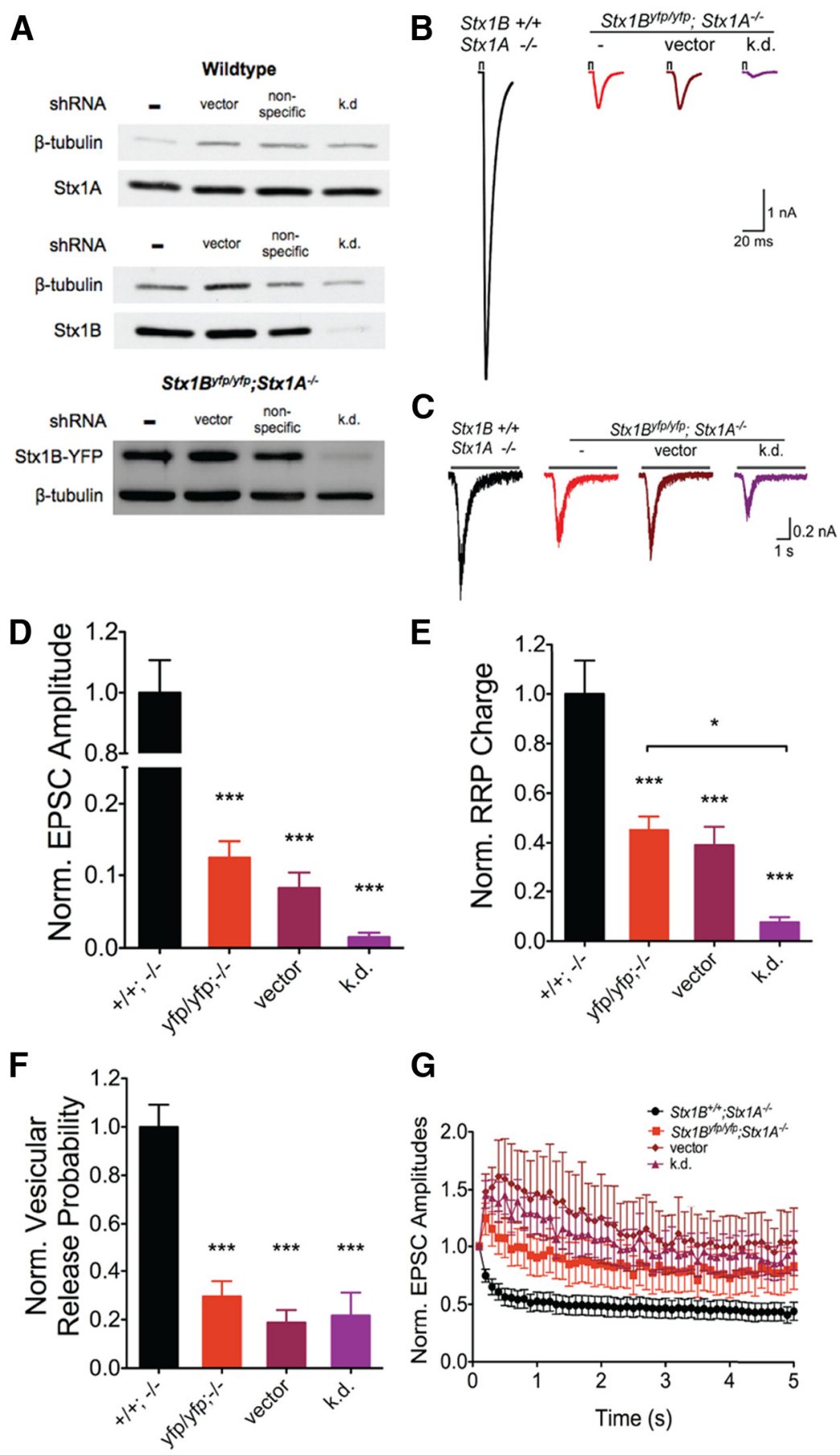

Figure 6. Knockdown of Stx1B-YFP further impaired release efficiency. $A$, Immunoblot showing knockdown of both Stx $1 B$ (in

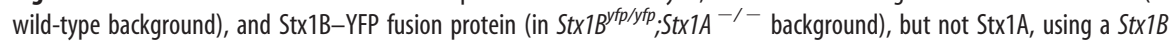
sequence-specific shRNA. B, Sample traces of EPSCs from Stx $1 B^{+/+} ;$Stx $1 A^{-/-}$(black), Stx $1 B^{\text {yfp/yfp }} ;$ Stx $1 A^{-1-}$ untransduced (red), transduced with lentiviral vector only (Lois et al., 2002), and transduced with knock-down construct (purple). C, Sample traces of RRP released by hyperosmotic stimulation. $\boldsymbol{D}$, Normalized summary plot of EPSC amplitudes (*** $<0.0001$ ). $\boldsymbol{E}$, Normalized summary plot of RRP charge $\left({ }^{*} p<0.05,{ }^{* * *} p<0.001\right)$. $\boldsymbol{F}$, Normalized summary plot of vesicular release probability. $\boldsymbol{G}$ Short-term plasticity behavior during $10 \mathrm{~Hz}$ stimulation.

vesicles; 62/0.5 $\mathrm{mm}^{2} ; \mathrm{SD}, 22 / 0.5 \mu \mathrm{m}^{2} ; F_{(3,116)}=5.3, p=$ 0.0018 and $F_{(3,116)}=7.3, p=0.0001$; Fig. $\left.9 E, F\right)$.

Priming and release probability as a function of Stx 1 expression levels

Different mechanisms can explain the regulation of release by Stx1, including clustering of Stx 1 molecules in large copy num- 
A
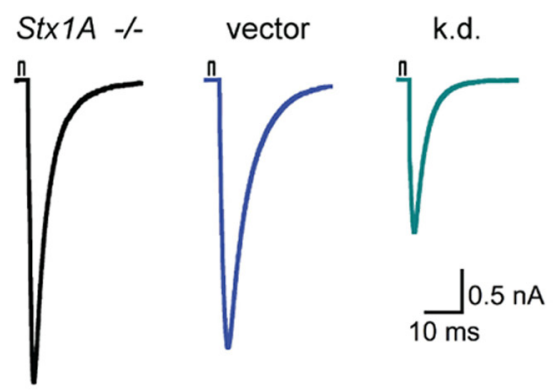

C

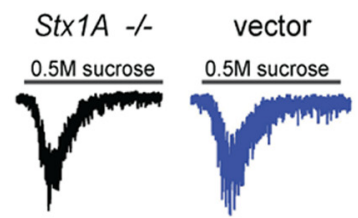

E

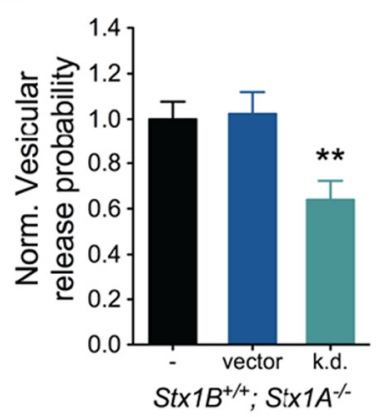

G
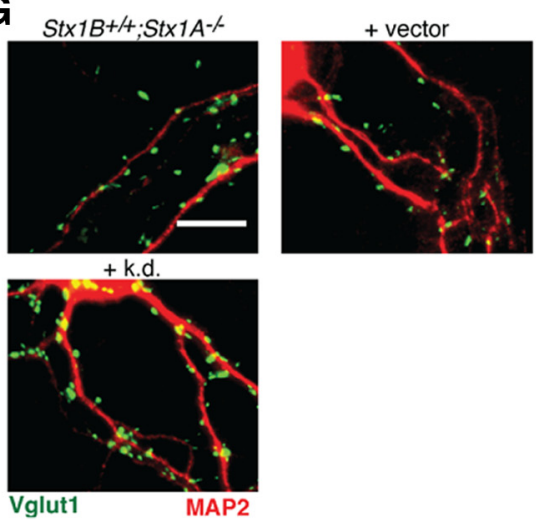

\section{$\mathbf{F}$}

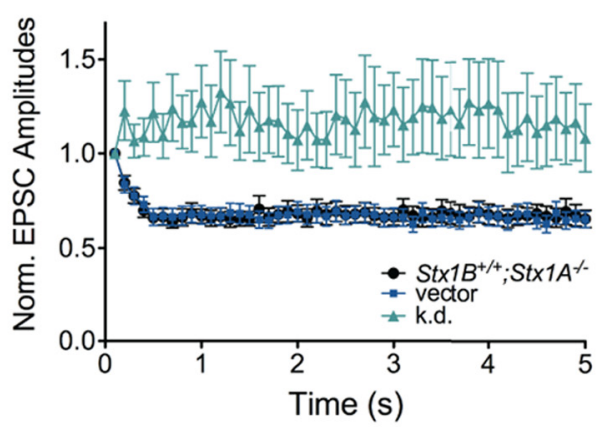

H

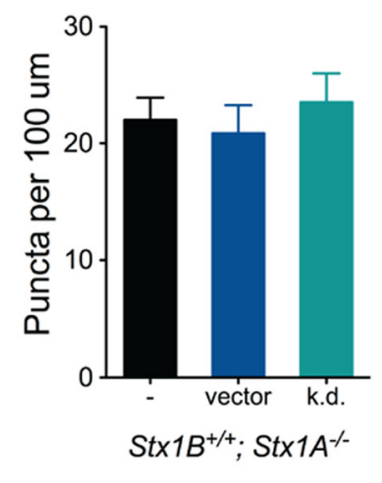

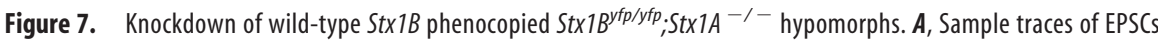
from Stx $1 B^{+/+} ;$Stx $1 A^{-/-}$neurons untransduced (black), transduced with lentiviral vector only (blue), or transduced with a Stx1B sequence-specific shRNA (green). $\boldsymbol{B}$, Normalized summary plot of EPSC amplitudes ( $\left.{ }^{* * *} p<0.0001\right)$. $\boldsymbol{C}$, Sample traces of RRP released by hyperosmotic stimulation. $\boldsymbol{D}$, Normalized summary plot of RRP charge $\left({ }^{* * *} p<0.0001\right)$. $\boldsymbol{E}$, Normalized summary plot of vesicular release probability $\left({ }^{* *} p<0.001\right)$. $\boldsymbol{F}$, Short-term plasticity behavior during $10 \mathrm{~Hz}$ stimulation. G, Sample images of autaptic neurons double labeled with the synaptic marker VGluT1 and the dendritic marker MAP2. Scale bar, $10 \mu \mathrm{m}$. $\boldsymbol{H}$, Summary plot of synaptic vesicle density measured as VGluT1 puncta per $100 \mu \mathrm{m}$ of dendrite length.

bers around release sites (Barg et al., 2010; Knowles et al., 2010) and formation of Stx1:SNAP25 heterodimers that serve as acceptor complexes for Syb2 (Fasshauer and Margittai, 2004; Weninger et al., 2008). Since we have means to vary levels of Stxl over a range of concentrations that differentially affect vesicle priming and fusion efficiency, we used this as an opportunity to gain insights into the underlying process by performing quantitative analysis of function versus SNARE protein concentration. First, quantitative immunocytochemistry of Stx1 at synapses was performed, as it allowed us to determine the concentration directly at the site of release and provided us with built-in normalization procedures by costaining for a synaptic protein that is similarly anchored to the plasma membrane. Autaptic neurons were double labeled with antibodies against Stx1B and the active zone marker Bassoon (tom Dieck et al., 1998). Stx1B is expressed abundantly and has a patchy staining pattern throughout the neuron, while Bassoon has a punctate staining pattern that marks release sites (Fig. 10C). Stx1B expression levels were measured as fluorescence intensity only in regions marked by Bassoon and then were normalized to the intensity levels of the marker. There were no significant differences in mean fluorescence intensity of Bassoon among the groups $\left(\right.$ Stx $1 B^{+/+}$; Stx $1 A^{-/-}$fluorescence: 1647 units; SD, 288.4; Stx $1 B^{+/ y f p} ;$ Stx $1 A^{-/-}$fluorescence: 2980 units; SD, 577.9; Stx1B $B^{+/+} ;$Stx $1 A^{-/-}$ + k.d. fluorescence: 1791 units; SD, 203.9; Stx $1 B^{y f p / y f p} ;$ Stx $1 A^{-/-}$fluorescence: 2244 units; SD, 300.3; Stx $1 B^{y f p / y f p} ;$ Stx $1 A^{-/-}+$ k.d. fluorescence: 2972 units; SD, 450.5; $F_{(5,55)}=1.538, p=0.2056$; Fig. 10B). Hypomorphic expression of Stx1B, combined with knockdown of either wild-type Stx1 or Stx1B-YFP, resulted in graded reductions in Stx1 expression $\left(\right.$ Stx $1 B^{+/+}$; Stx $1 A^{-/-}$fluorescence: 376.7 ; SD, 83.36; Stx $1 B^{+/ y f p} ;$ St $x 1 A^{-/-}$fluorescence: 288.6 ; SD, 38.75; Stx $1 B^{+/+} ;$Stx $1 A^{-/-}+$k.d. fluorescence: 77.89 ; SD, 17.73; Stx $1 B^{y f p / y f p}$; Stx1A $1 A^{-/-}$fluorescence: 135.3; SD, 27.13; Stx $1 B^{y f p / y f p} ;$ Stx $1 A^{-1-}+$ k.d. fluorescence: 84.82; SD, 22.68; $F_{(5,56)}=8.737, p<0.0001$; Fig. 10A). To account for trial-to-trial variability, fluorescence intensities were normalized to the mean fluorescence intensities of Stx $1 B^{+/+} ; S t x 1 A^{-/-}$neurons per culture. Normalized Stx1B/Bassoon ratios were then calculated for a more precise quantification of the graded reductions $\left(S t \times 1 B^{+/+}\right.$; Stx $1 A^{-/-}$with knock-down ratio: 0.16 ; SD, 0.16; Stx $1 B^{y f p / y f p} ;$ Stx $1 A^{-1-}$ ratio: 0.15; SD, 0.11 ; Stx $1 B^{y f p / y f p} ;$ Stx $1 A^{-/-}$with knock- 
down ratio: 0.09; $\mathrm{SD}, 0.07$; with respect to Stx $1 B^{+/+} ;$Stx $1 A^{-/-}$controls; $F_{(4,51)}=11.3$, $p<0.0001$; Fig. 10D).

To measure how RRP size and $P_{\mathrm{vr}}$ change with increasing Stx1 levels, previously calculated values from different experimental groups were then normalized and plotted as a function of Stx $1 B$ expression (Fig. $10 E, F$ ) and were fitted with the standard Hill equation (see Materials and Methods). The mean k.d. for syntaxin was similar for priming and $P_{\mathrm{vr}}$, with values 0.19 -fold and 0.18 -fold of wild-type expression, respectively. In addition, the Hill coefficient or cooperativity was 3.4 for RRP size and 2.5 for $P_{\mathrm{vr}}$.

At expression levels between $10 \%$ and $50 \%$ of wild type, small changes in Stxl concentration lead to steep changes in RRP size and $P_{\mathrm{vr}}$ (Fig. $10 E, F$ ). These data suggest that at very low concentrations, Stx1 is a rate-limiting factor for priming and release efficiency. In addition, our estimates suggest that the Stx1 "cooperative unit" for both RRP size and vesicular release probability is $\sim 3$, implying that priming and vesicle fusion have the same molecular stoichiometry.

\section{Discussion}

Although the function of SNARE proteins in membrane fusion has been well characterized, their roles at distinct steps of the synaptic vesicle cycle, such as docking and priming, are poorly understood. Our main objective was to investigate how manipulation of SNARE protein expression levels affect docking, priming, and release probability. We used a unique experimental approach that diminishes neurotransmitter release by controlled genetic reduction of Stx 1 expression in mammalian central synapses. Two methods were used, either individually or in combination, to reduce Stxl gene expression in hippocampal neurons in a graded fashion. First, we took advantage of a $S t x 1 B^{y f p}$ knock-in mouse line that expresses an Stx1B-YFP fusion protein in a hypomorphic fashion. Second, we used RNAimediated knockdown to reduce wild-type Stx 1 and Stx1-YFP expression levels. This complementary approach offers two advantages: a means to check for artifacts, and multiple data points for analyzing the relationship between reduction of Stxl expression and neurotransmitter release.

Our results lead us to several conclusions. First, due to the observation that a severe reduction in $S t x 1 B$ expression alone leads to juvenile lethality in mice, we conclude that mammalian Stx $1 B$, in contrast to Stx $1 A$, has an essential role in postnatal development. Both Stxl isoforms are coexpressed abundantly throughout the adult rodent nervous system, though there is evidence of differential distribution and enrichment of one isoform
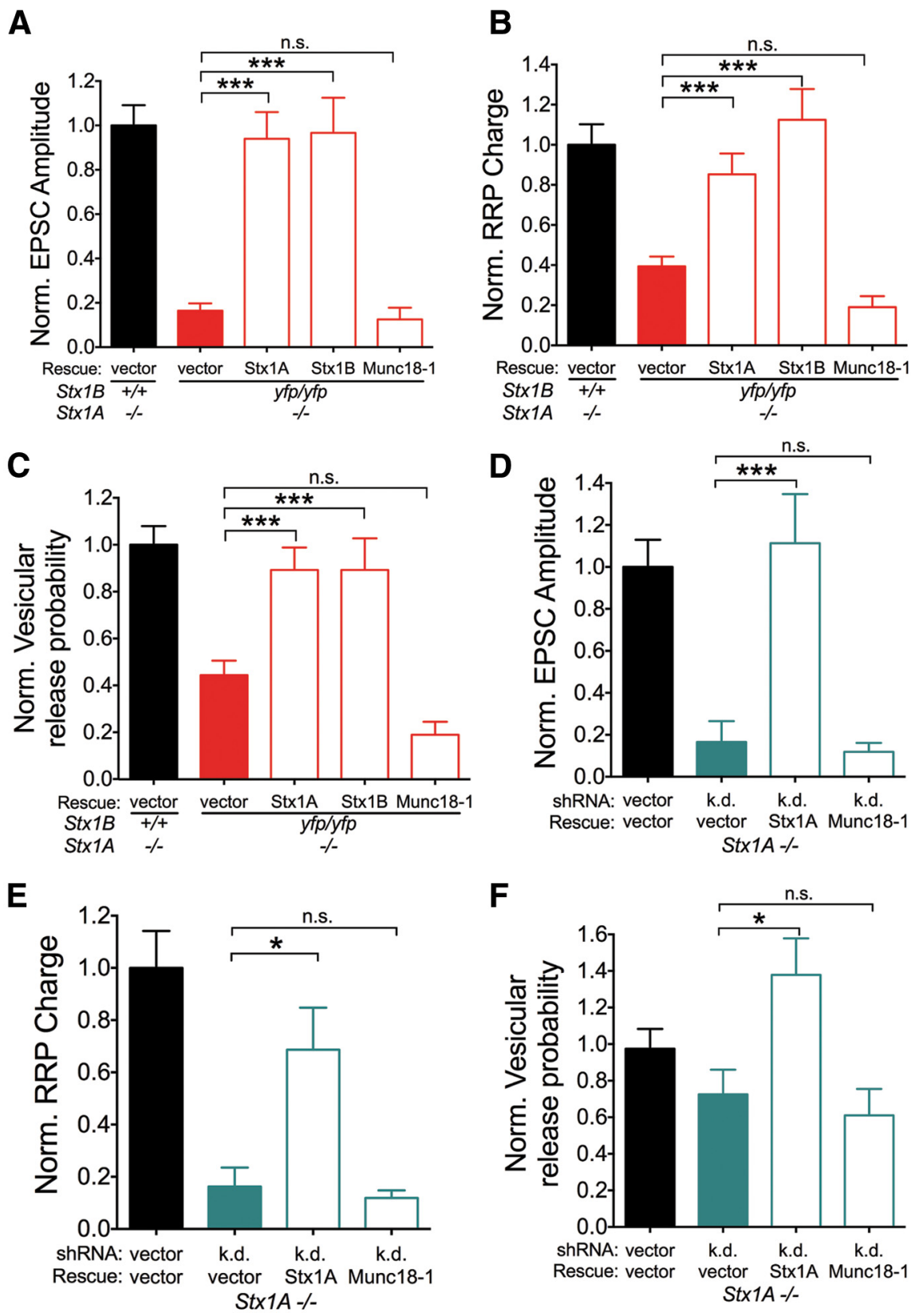

Figure 8. Lentiviral expression of either Stx1A or Stx1B, but not Munc18-1, rescues synaptic release in neurons with severe reduction in Stx1 levels. $\boldsymbol{A}-\boldsymbol{C}$, Normalized summary plots of EPSC peak amplitudes $(\boldsymbol{A})$, RRP charge $(\boldsymbol{B})$, and vesicular release probability $(\boldsymbol{C})$ in control Stx $1 B^{\text {yfp/yfp. }} ;$ Stx $1 A^{-\prime-}$ neurons (black), and Stx $1 B^{y f p / y f p} ;$ Stx $1 A^{-/-}$neurons transduced without (red) or with Stx1A, Stx1B, or Munc18-1 rescue constructs (red outlines; ANOVA with Tukey's test, ${ }^{* * *} p<0.001 ;$ n.s., not significant). $\boldsymbol{D}-\boldsymbol{F}$, Normalized summary plots of EPSC peak amplitudes $(\boldsymbol{D}), \operatorname{RRP}$ charge $(\boldsymbol{E})$, and vesicular release probability $(\boldsymbol{F})$ in Stx $1 A^{-/-}$neurons without (black) or with shRNA-mediated knockdown of Stx1B (teal), and knockdown neurons transduced with Stx $1 A$, Stx 1B, or Munc18-1 rescue constructs (teal outlines; ANOVA with Tukey's test, ${ }^{*} p<0.05,{ }^{* * *} p<0.001$; n.S., not significant).

over the other in certain regions (Ruiz-Montasell et al., 1996; Aguado et al., 1999). Since behavioral analysis was not performed, we are not able to comprehensively determine which specific neuronal populations are dysfunctional in $S t x 1 B^{y f p / y f p}$ mice.

The possibility that the Stx1B-YFP fusion protein has a neomorphic effect is not likely for several reasons. First, the YFP tag is localized extracellularly, indicating that the fluorophore is not in a position to sterically hinder SNARE function. Second, the Stx1B-YFP allele does not have apparent dominant-negative effects, as indicated by the lack of significant changes in evoked 

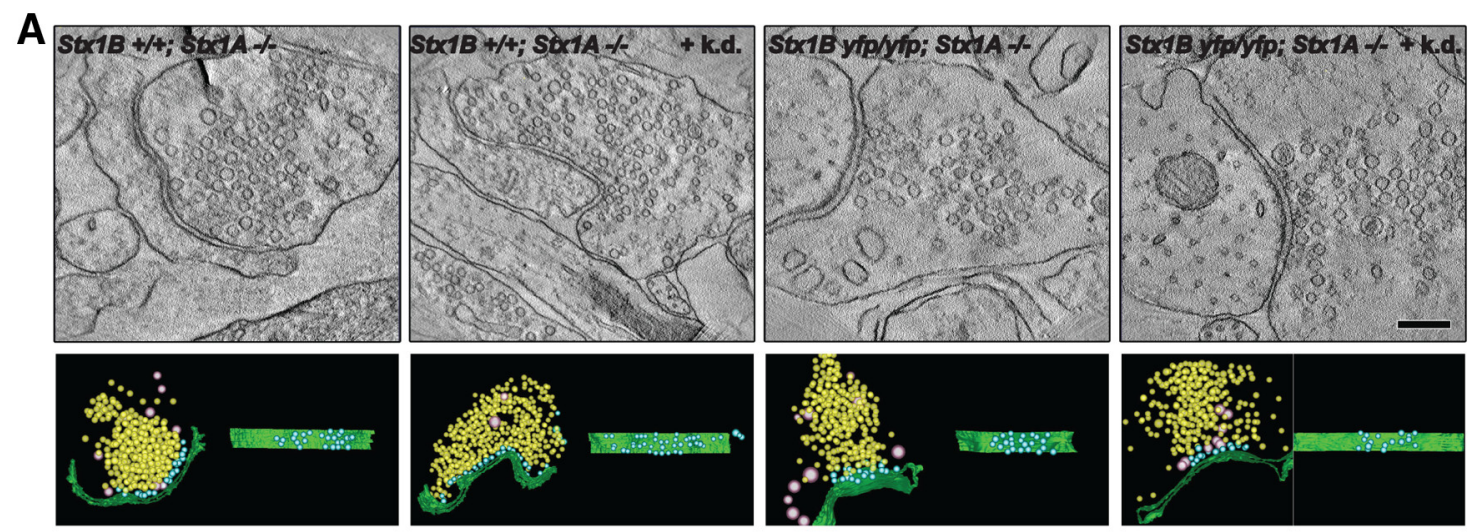
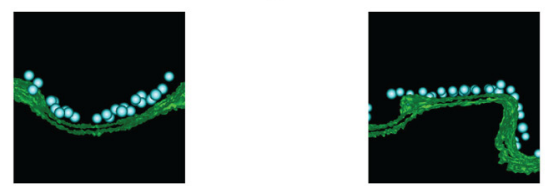

B
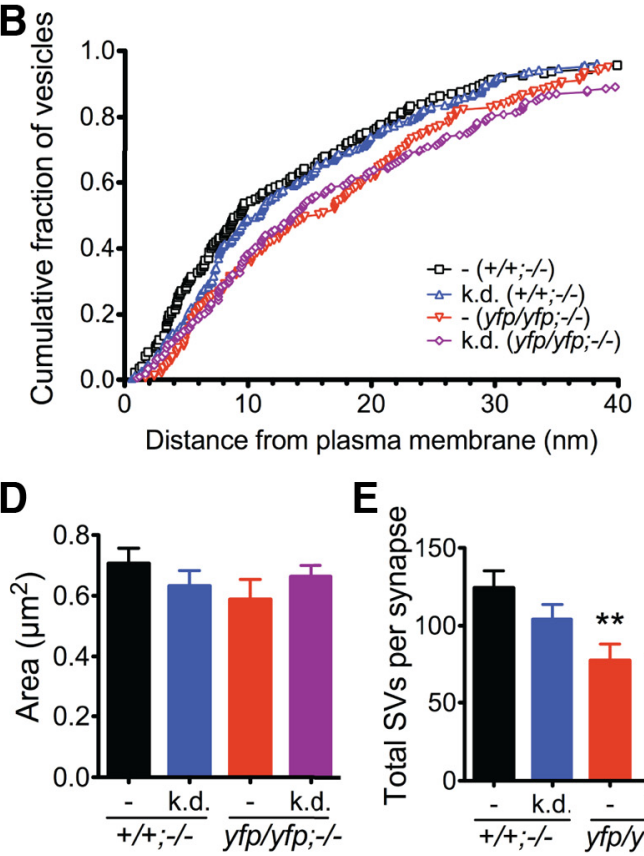

E。

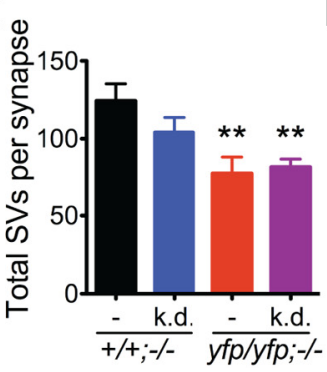

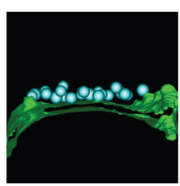

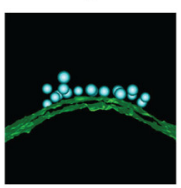

C

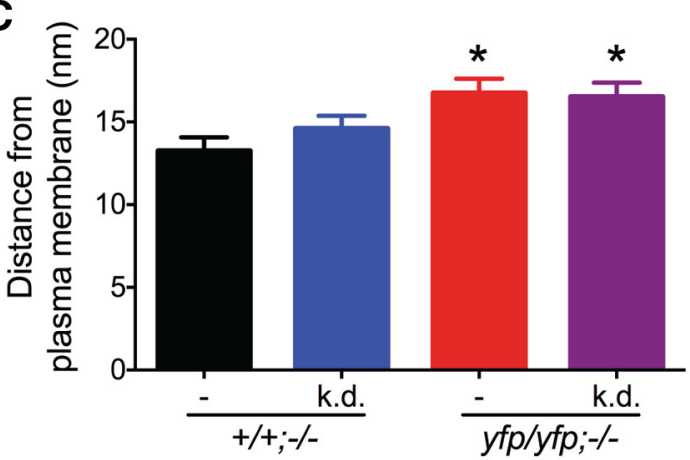

F

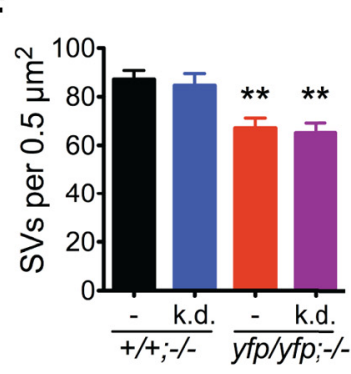

G

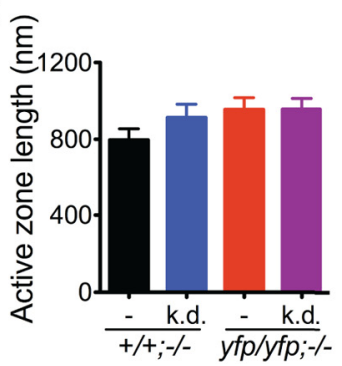

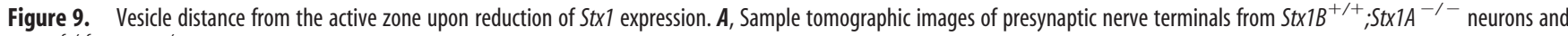
Stx $1 B^{\text {yfp/yfp; }}$ Stx $1 A^{-1-}$ neurons that were untransduced and transduced with a Stx $1 B$ sequence-specific shRNA. Scale bar, $100 \mathrm{~nm}$. $B$, Cumulative distribution of vesicle distance from the plasma membrane. C, Summary plot of vesicle distance within $40 \mathrm{~nm}$ of the plasma membrane. ${ }^{*} p<0.05$. D, Summary plot of presynaptic area. $\boldsymbol{E}$, Summary plot of total number of synaptic vesicles per synapse. ${ }^{* *} p<0.001$. F, Summary plot of synaptic vesicle density (ANOVA with Tukey's test, ${ }^{* *} p<0.001$ ). G, Summary plot of active zone length.

release amplitudes, spontaneous release frequency, RRP size, and $P_{\mathrm{vr}}$ of $S t x 1 B^{+/ y f p} ;$ Stx $1 A^{-/-}$neurons compared with controls and Stx $1 B^{y f p / y f p} ;$ Stx $1 A^{+/+}$neurons compared with wild type. Third, Stx1 knock-down phenotypes lead to similar decreases in EPSC amplitude, RRP size, and $P_{\mathrm{vr}}$, providing complementary evidence that severe reductions in Stx1 levels impair release efficiency.

Severe reductions in total Stx 1 expression are needed to impair neurotransmitter release in central synapses. First, reduction of Stx1B levels alone does not impair neurotransmission. Only in the absence of Stx1A expression does the reduction of Stx1B in hippocampal neurons (either by knockdown or hypomorphic expression of $S t x 1 B$ in hippocampal neurons) diminish evoked and spontaneous forms of release. Also, Stx $1 B^{+/ y f p} ;$ Stx $1 A^{-1-}$ heterozygotes do not show any significant changes in neurotransmitter release, supporting the notion that expressing $S t x 1 B$ at levels below $\sim 50 \%$ of wild type are needed to induce a decline in priming and $P_{\mathrm{vr}}$.

Previous studies on reduced levels of SNARE or SNAREassociated proteins have shown sensitivity similar to those in our findings. On the mRNA level, reduction of SNAP25 of $>85 \%$ does not produce a measurable change in synaptic transmission in cultured neurons (Sharma et al., 2011). Analyses of hypomorphic syntaxin-1 alleles in Drosophila and Caenorhabditis elegans (Schulze et al., 1995; Saifee et al., 1998) yielded distinct severities of phenotypes, though the relationship of syntaxin levels with docking, priming, and release probability was not analyzed.

We observed a relatively selective corresponding decrease in Munc18-1 levels upon reduction of Stx1B expression levels. The inverse situation, which involves the decrease of Stx1A and Stx1B levels in the absence of Munc18-1, has been observed in previous 
studies (Toonen et al., 2005). Thus, Stx1 and Munc18-1 are chaperones of each other-deficiency in one leads to instability in the other. This relationship has a functional significance, as demonstrated by overexpression studies in which inhibition of neurotransmission by Stxl overexpression was suppressed by cooverexpression of Munc18-1 and vice versa (Wu et al., 1998; Mitchell and Ryan, 2005). These findings suggest that the synaptic phenotypes we observed upon reduction of Stxl levels are due to the associated loss of Munc18-1. However, we did not observe any significant rescue of EPSC amplitudes, RRP, and $P_{\mathrm{vr}}$ in Stx1-deficient neurons transduced only with Munc18-1 (Fig. 8), excluding this possibility.

Fourth, we found similar sensitivity and a strong cooperative unit of $\sim 3$ for both Stx1-dependent priming and release probability, suggesting that these two processes arise from the same molecular process.

Our results on docking are different from earlier studies, which found no significant changes in docked vesicles upon expression of BoNT/C in neurons (de Wit et al., 2006, 2009). The involvement of mammalian Stx1 in vesicle docking has been suggested before by total internal reflection fluorescence analysis of PC12 cells, which showed that Stx1 and SNAP25 cluster in large copy numbers at sites where secretory granules dock (Barg et al., 2010; Knowles et al., 2010). Whether large clusters of SNARE proteins are actively involved in the docking process or simply increase the likelihood of SNARE complex formation is not clear. Our findings show that even with a significant reduction of Stx1 expression in knock-down neurons, the change in vesicle distribution is relatively subtle, suggesting that the former mechanism is unlikely.

Acute interference of Stx 1 function in the calyx of Held by botulinum toxin cleavage blocked neurotransmitter release without changing the kinetics of the vesicle exocytosis (Sakaba et al., 2005). This experiment confirmed that Stx1 is important, but since it creates a soluble Stxl fragment, its interpretation is not unequivocal. Here, we show that the vesicular release probability in central synapses was affected by reduction of Stx1, suggesting that SNARE proteins are rate limiting for $\mathrm{Ca}^{2+}$-evoked release, provided their concentration has decreased below a certain minimal level. From a mechanistic perspective, a decrease in release probability is likely due to the availability of fewer Stx 1 molecules that can form functional SNARE complexes, which can decrease the driving force for fusion. Previous estimates for the minimum number of SNARE complexes required for fusion ranged from 5 to 11 (Han et al., 2004; Domanska et al., 2009; Karatekin et al., 2010), favoring the formation of multimeric SNARE complexes per vesicle to facilitate exocytosis. Other studies, however, have challenged the multimeric model by arriving at lower estimates of
B

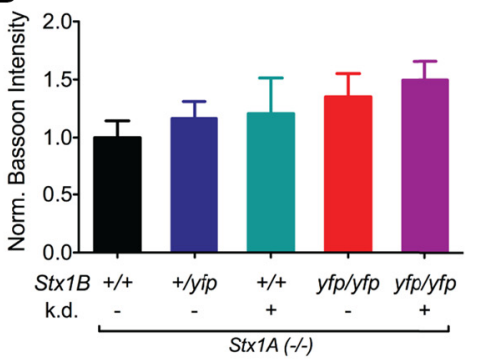

D

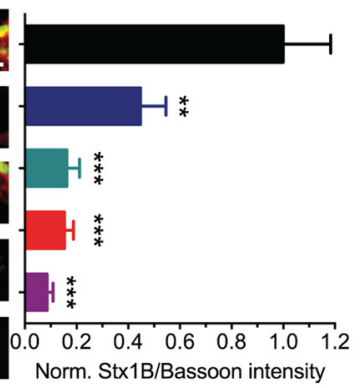

$\mathbf{F}$

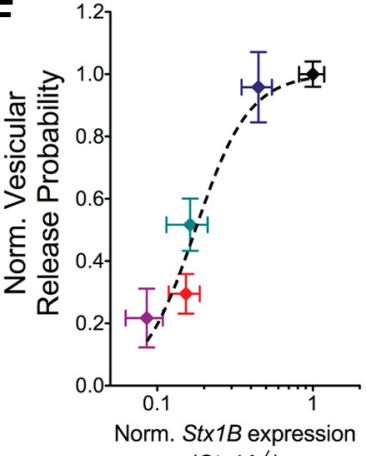

$\left(S t \times 1 A^{-/-}\right)$
- $S t \times 1 B^{+/+} ; S t \times 1 A^{-/-}$ -Stx1B+/yfp;Stx1A -Stx1 $1 B^{+/+} ;$Stx $1 A^{-/-}+$k.d. -Stx1Byfp/yfp;Stx1A-/-Stx1Byfp/yfp;Stx1A-/- + k.d.

Figure 10. Priming and release probability as a function of Stx 1 expression levels. $\boldsymbol{A}$, Normalized summary plot of Stx $1 B$ fluorescence intensities. ${ }^{* *} p<0.0001$. B, Normalized summary plot of Bassoon fluorescence intensities. C, Sample images from double labeling of autaptic neurons with anti-Stx1B and anti-Bassoon antibodies. Scale bar, $5 \mu \mathrm{m}$. D, Summary plot of Stx1B/ vesicular release probability $(\boldsymbol{F})$, derived from previously shown data, were plotted as a function of normalized Stx $1 B$ expression

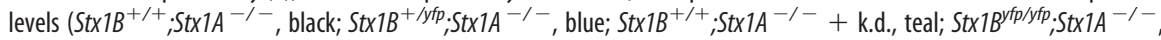

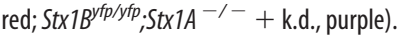

two to three in live cells (Hua and Scheller, 2001; Mohrmann et al., 2010; Sinha et al., 2011), and one SNARE complex using liposome fusion assays (van den Bogaart et al., 2010). We estimated a Stx 1 cooperative unit of 3 for both priming and release probability. Taken collectively with studies involving SNAP25 (Mohrmann et al., 2010) and Syb2 (Sinha et al., 2011), there is now evidence from all three SNARE proteins that efficient exocytosis in vivo generally requires more than one SNARE complex. Severely decreasing the number of SNARE complexes per vesicle reduces release efficiency, though we cannot exclude the possibility that fusion can occur with a single SNARE complex.

Having the same cooperativity for priming a vesicle and for the regulation of the release probability has interesting biological implications for SNARE-mediated fusion. It has been proposed that molecular priming of a vesicle involves partial assembly of the SNARE complex at the $\mathrm{N}$ terminus, and that full assembly toward the $\mathrm{C}$ terminus upon $\mathrm{Ca}^{2+}$ influx facilitates fusion (Südhof, 1995; Sørensen et al., 2006; Walter et al., 2010). Our findings that docking, priming, and vesicular release probability are all 
affected upon severe Stx1 reduction, with an accompanying decrease in Munc18-1 levels, support the idea that they are highly related processes. At the very least, our results imply that molecular priming is a direct regulation of release probability, via modulation of vesicle fusogenicity. This goes against the suggestion that SNARE complex formation only occurs downstream of calcium influx (van den Bogaart et al., 2011). A cooperative unit of $\sim 3$ provides a minimum estimate of Stx1 molecules needed for these processes, presumably to form functional SNARE complexes between vesicles and release sites, corresponding to molecular priming. Primed vesicles attain fusogenicity, enabling them to be released spontaneously, by an action potential or by hyperosmotic stimulus. Consistent with this idea, the approximate fit of the $P_{\mathrm{vr}}$ function approaches zero at very low Stxl concentrations (Fig. 10F). Fusion efficiency as a function of Stx1 levels will be difficult to assess at very low concentrations, as the small response size sets a threshold for detection $P_{\mathrm{vr}}$ values of $\sim 1 \%$. Nevertheless, our results suggest that SNARE proteins are rate limiting, and that we need more than one SNARE complex for fusion.

Our model proposes that small changes in the number of SNARE complexes can regulate release by affecting the number of vesicles reaching the threshold for fusogenicity. This, in turn, affects how likely they will be released by an action potential. In the same way, molecules such as Munc13 (Basu et al., 2007) and complexin (Xue et al., 2007, 2010) can also regulate $P_{\mathrm{vr}}$, through conformational changes or direct interactions that enhance the performance of the fusion machinery. These mechanisms are distinct from $\mathrm{Ca}^{2+}$-dependent modulation of release, such as the one mediated by synaptotagmin 1 (Fernández-Chacón et al., 2001; Dai et al., 2007; Xue et al., 2008).

\section{References}

Aguado F, Majó G, Ruiz-Montasell B, Llorens J, Marsal J, Blasi J (1999) Syntaxin $1 \mathrm{~A}$ and $1 \mathrm{~B}$ display distinct distribution patterns in the rat peripheral nervous system. Neuroscience 88:437-446. CrossRef Medline

Barg S, Knowles MK, Chen X, Midorikawa M, Almers W (2010) Syntaxin clusters assemble reversibly at sites of secretory granules in live cells. Proc Natl Acad Sci U S A 107:20804-20809. CrossRef Medline

Basu J, Betz A, Brose N, Rosenmund C (2007) Munc13-1 C1 domain activation lowers the energy barrier for synaptic vesicle fusion. J Neurosci 27:1200-1210. CrossRef Medline

Bennett MK, Calakos N, Scheller RH (1992) Syntaxin: a synaptic protein implicated in docking of synaptic vesicles at presynaptic active zones. Science 257:255-259. CrossRef Medline

Chao HT, Zoghbi HY, Rosenmund C (2007) MeCP2 controls excitatory synaptic strength by regulating glutamatergic synapse number. Neuron 56:58-65. CrossRef Medline

Clements JD, Bekkers JM (1997) Detection of spontaneous synaptic events with an optimally scaled template. Biophys J 73:220-229. CrossRef Medline

Dai H, Shen N, Araç D, Rizo J (2007) A quaternary SNARE-synaptotagmin$\mathrm{Ca} 2+$-phospholipid complex in neurotransmitter release. J Mol Biol 367: 848-863. CrossRef Medline

Delgado-Martínez I, Nehring RB, Sørensen JB (2007) Differential abilities of SNAP-25 homologs to support neuronal function. J Neurosci 27:93809391. CrossRef Medline

de Wit H, Cornelisse LN, Toonen RF, Verhage M (2006) Docking of secretory vesicles is syntaxin dependent. PLoS One 1:e126. CrossRef Medline

de Wit H, Walter AM, Milosevic I, Gulyás-Kovács A, Riedel D, Sørensen JB, Verhage M (2009) Synaptotagmin-1 docks secretory vesicles to syntaxin-1/SNAP-25 acceptor complexes. Cell 138:935-946. CrossRef Medline

Domanska MK, Kiessling V, Stein A, Fasshauer D, Tamm LK (2009) Single vesicle millisecond fusion kinetics reveals number of SNARE complexes optimal for fast SNARE-mediated membrane fusion. J Biol Chem 284: 32158-32166. CrossRef Medline

Dulubova I, Sugita S, Hill S, Hosaka M, Fernandez I, Südhof TC, Rizo J
(1999) A conformational switch in syntaxin during exocytosis: role of munc18. EMBO J 18:4372-4382. CrossRef Medline

Fasshauer D, Margittai M (2004) A transient N-terminal interaction of SNAP-25 and syntaxin nucleates SNARE assembly. J Biol Chem 279: 7613-7621. CrossRef Medline

Fernández-Chacón R, Königstorfer A, Gerber SH, García J, Matos MF, Stevens CF, Brose N, Rizo J, Rosenmund C, Südhof TC (2001) Synaptotagmin I functions as a calcium regulator of release probability. Nature 410 : 41-49. CrossRef Medline

Fujiwara T, Mishima T, Kofuji T, Chiba T, Tanaka K, Yamamoto A, Akagawa K (2006) Analysis of knock-out mice to determine the role of HPC-1/ syntaxin 1A in expressing synaptic plasticity. J Neurosci 26:5767-5776. CrossRef Medline

Gerber SH, Rah JC, Min SW, Liu X, de Wit H, Dulubova I, Meyer AC, Rizo J, Arancillo M, Hammer RE, Verhage M, Rosenmund C, Südhof TC (2008) Conformational switch of syntaxin- 1 controls synaptic vesicle fusion. Science 321:1507-1510. CrossRef Medline

Han X, Wang CT, Bai J, Chapman ER, Jackson MB (2004) Transmembrane segments of syntaxin line the fusion pore of $\mathrm{Ca} 2+$-triggered exocytosis. Science 304:289-292. CrossRef Medline

Hua Y, Scheller RH (2001) Three SNARE complexes cooperate to mediate membrane fusion. Proc Natl Acad Sci U S A 98:8065-8070. CrossRef Medline

Jahn R, Scheller RH (2006) SNAREs—engines for membrane fusion. Nat Rev Mol Cell Biol 7:631-643. CrossRef Medline

Karatekin E, Di Giovanni J, Iborra C, Coleman J, O’Shaughnessy B, Seagar M, Rothman JE (2010) A fast, single-vesicle fusion assay mimics physiological SNARE requirements. Proc Natl Acad Sci U S A 107:3517-3521. CrossRef Medline

Kim JH, Lee SR, Li LH, Park HJ, Park JH, Lee KY, Kim MK, Shin BA, Choi SY (2011) High cleavage efficiency of a 2A peptide derived from porcine teschovirus-1 in human cell lines, zebrafish and mice. PLoS One 6:e18556. CrossRef Medline

Knowles MK, Barg S, Wan L, Midorikawa M, Chen X, Almers W (2010) Single secretory granules of live cells recruit syntaxin- 1 and synaptosomal associated protein 25 (SNAP-25) in large copy numbers. Proc Natl Acad Sci U S A 107:20810-20815. CrossRef Medline

Lois C, Hong EJ, Pease S, Brown EJ, Baltimore D (2002) Germline transmission and tissue-specific expression of transgenes delivered by lentiviral vectors. Science 295:868-872. CrossRef Medline

Mitchell SJ, Ryan TA (2005) Munc18-dependent regulation of synaptic vesicle exocytosis by syntaxin-1A in hippocampal neurons. Neuropharmacology 48:372-380. CrossRef Medline

Mohrmann R, de Wit H, Verhage M, Neher E, Sørensen JB (2010) Fast vesicle fusion in living cells requires at least three SNARE complexes. Science 330:502-505. CrossRef Medline

Ogawa H, Harada S, Sassa T, Yamamoto H, Hosono R (1998) Functional properties of the unc-64 gene encoding a Caenorhabditis elegans syntaxin. J Biol Chem 273:2192-2198. CrossRef Medline

Rizo J, Südhof TC (2012) The membrane fusion enigma: SNAREs, Sec1/ Munc18 proteins, and their accomplices—guilty as charged? Annu Rev Cell Dev Biol 28:279-308. CrossRef Medline

Rosenmund C, Stevens CF (1996) Definition of the readily releasable pool of vesicles at hippocampal synapses. Neuron 16:1197-1207. CrossRef Medline

Rosenmund C, Feltz A, Westbrook GL (1995) Synaptic NMDA receptor channels have a low open probability. J Neurosci 15:2788-2795. Medline

Ruiz-Montasell B, Aguado F, Majó G, Chapman ER, Canals JM, Marsal J, Blasi J (1996) Differential distribution of syntaxin isoforms 1A and 1B in the rat central nervous system. Eur J Neurosci 8:2544-2552. CrossRef Medline

Saifee O, Wei L, Nonet ML (1998) The Caenorhabditis elegans unc-64 locus encodes a syntaxin that interacts genetically with synaptobrevin. Mol Biol Cell 9:1235-1252. CrossRef Medline

Sakaba T, Stein A, Jahn R, Neher E (2005) Distinct kinetic changes in neurotransmitter release after SNARE protein cleavage. Science 309:491-494. CrossRef Medline

Schoch S, Deák F, Königstorfer A, Mozhayeva M, Sara Y, Südhof TC, Kavalali ET (2001) SNARE function analyzed in synaptobrevin/VAMP knockout mice. Science 294:1117-1122. CrossRef Medline

Schulze KL, Broadie K, Perin MS, Bellen HJ (1995) Genetic and electrophysiological studies of Drosophila syntaxin-1A demonstrate its role in non- 
neuronal secretion and neurotransmission. Cell 80:311-320. CrossRef Medline

Sharma M, Burré J, Südhof TC (2011) CSPalpha promotes SNAREcomplex assembly by chaperoning SNAP-25 during synaptic activity. Nat Cell Biol 13:30-39. CrossRef Medline

Sinha R, Ahmed S, Jahn R, Klingauf J (2011) Two synaptobrevin molecules are sufficient for vesicle fusion in central nervous system synapses. Proc Natl Acad Sci U S A 108:14318-14323. CrossRef Medline

Sørensen JB, Wiederhold K, Muller EM, Milosevic I, Nagy G, de Groot BL, Grubmuller H, Fasshauer D (2006) Sequential N- to C-terminal SNARE complex assembly drives priming and fusion of secretory vesicles. EMBO J 25:955-966. CrossRef Medline

Südhof TC (1995) The synaptic vesicle cycle: a cascade of protein-protein interactions. Nature 375:645-653. CrossRef Medline

Südhof TC (2004) The synaptic vesicle cycle. Annu Rev Neurosci 27:509547. CrossRef Medline

Südhof TC, Rothman JE (2009) Membrane fusion: grappling with SNARE and SM proteins. Science 323:474-477. CrossRef Medline

tom Dieck S, Sanmartí-Vila L, Langnaese K, Richter K, Kindler S, Soyke A, Wex H, Smalla KH, Kämpf U, Fränzer JT, Stumm M, Garner CC, Gundelfinger ED (1998) Bassoon, a novel zinc-finger CAG/glutaminerepeat protein selectively localized at the active zone of presynaptic nerve terminals. J Cell Biol 142:499-509. CrossRef Medline

Toonen RF, de Vries KJ, Zalm R, Südhof TC, Verhage M (2005) Munc18-1 stabilizes syntaxin 1 , but is not essential for syntaxin 1 targeting and SNARE complex formation. J Neurochem 93:1393-1400. CrossRef Medline

van den Bogaart G, Holt MG, Bunt G, Riedel D, Wouters FS, Jahn R (2010) One SNARE complex is sufficient for membrane fusion. Nat Struct Mol Biol 17:358-364. CrossRef Medline

van den Bogaart G, Thutupalli S, Risselada JH, Meyenberg K, Holt M, Riedel
D, Diederichsen U, Herminghaus S, Grubmuller H, Jahn R (2011) Synaptotagmin-1 may be a distance regulator acting upstream of SNARE nucleation. Nat Struct Mol Biol 18:805-812. CrossRef Medline

Walter AM, Wiederhold K, Bruns D, Fasshauer D, Sørensen JB (2010) Synaptobrevin N-terminally bound to syntaxin-SNAP-25 defines the primed vesicle state in regulated exocytosis. J Cell Biol 188:401-413. CrossRef Medline

Washbourne P, Thompson PM, Carta M, Costa ET, Mathews JR, LopezBenditó G, Molnár Z, Becher MW, Valenzuela CF, Partridge LD, Wilson MC (2002) Genetic ablation of the t-SNARE SNAP-25 distinguishes mechanisms of neuroexocytosis. Nat Neurosci 5:19-26. CrossRef Medline

Weninger K, Bowen ME, Choi UB, Chu S, Brunger AT (2008) Accessory proteins stabilize the acceptor complex for synaptobrevin, the 1:1 syntaxin/SNAP-25 complex. Structure 16:308-320. CrossRef Medline

Wu MN, Littleton JT, Bhat MA, Prokop A, Bellen HJ (1998) ROP, the Drosophila Sec1 homolog, interacts with syntaxin and regulates neurotransmitter release in a dosage-dependent manner. EMBO J 17:127-139. CrossRef Medline

Xue M, Reim K, Chen X, Chao HT, Deng H, Rizo J, Brose N, Rosenmund C (2007) Distinct domains of complexin I differentially regulate neurotransmitter release. Nat Struct Mol Biol 14:949-958. CrossRef Medline

Xue M, Ma C, Craig TK, Rosenmund C, Rizo J (2008) The Janus-faced nature of the $\mathrm{C}(2) \mathrm{B}$ domain is fundamental for synaptotagmin-1 function. Nat Struct Mol Biol 15:1160-1168. CrossRef Medline

Xue M, Craig TK, Xu J, Chao HT, Rizo J, Rosenmund C (2010) Binding of the complexin $\mathrm{N}$ terminus to the SNARE complex potentiates synapticvesicle fusogenicity. Nat Struct Mol Biol 17:568-575. CrossRef Medline

Zhao C, Dreosti E, Lagnado L (2011) Homeostatic synaptic plasticity through changes in presynaptic calcium influx. J Neurosci 31:7492-7496. CrossRef Medline 